\title{
UNDRAINED SLIDING RESISTANCE OF SHALLOW FOUNDATIONS SUBJECT TO TORSION
}

\author{
Hamidreza Nouri ${ }^{1}$, Giovanna Biscontin ${ }^{2}$ and Charles P. Aubeny, M. ASCE ${ }^{3}$
}

\begin{abstract}
\end{abstract}
While the behavior of shallow foundations under vertical load combinations has been the subject of numerous studies, the response of shallow foundations subjected to combined horizontal and torsional loading has received considerably less attention. New offshore applications of shallow foundations for LNG facilities and other subsea structures have underscored the importance of the behavior of shallow embedded foundations subjected to combined in-plane translation and torsion. This study investigates the undrained bearing capacity of rectangular and square shallow foundations under eccentric horizontal loads through comparisons of various limit equilibrium and plastic limit analysis solutions to 3-D finite element solutions. In general, the plastic limit approach considered in this paper agrees well with the finite element solutions, although it has some tendency to over-predict capacity at greater embedment depths. The studies revealed a general insensitivity in the shape of the yield envelope to variations in embedment depth, which permits a simplified analysis suitable for first order estimates of load capacity. The variables considered in this study include footing aspect ratio, embedment depth, and load direction in addition to eccentricity.

KEY WORDS: shallow foundations, marine foundations, torsional resistance, sliding resistance, limit equilibrium, plastic limit analysis, finite element analysis, failure envelope.

\footnotetext{
${ }^{1}$ Project Geotechnical Engineer, Golder Associates, Inc., Redmond, WA, 98052

${ }^{2}$ University Lecturer in Geotechnical Engineering, University of Cambridge, Cambridge CB2 1PZ

${ }^{3}$ Professor, Texas A\&M University, College Station, TX 77843 (corresponding author). Email:caubeny@civil.tamu.edu
} 
27 Conventional methods of analyzing the bearing capacity of shallow foundations are mostly focused on the effect of moment and horizontal forces on vertical bearing capacity of the foundation, while the response of the foundation under torsion and combined sliding-torsion has received less attention. The increasing use of shallow foundations for offshore structures has underscored the significance of developing a better insight on the effect of torsion on sliding bearing capacity of foundations. Gravity based shallow foundations are widely used in offshore environments as they are commercially attractive options to support subsea infrastructure (Fisher and Cathie, 2002). In the offshore industry, Liquid Natural Gas (LNG) facilities, protection structures, pipeline end manifolds (PLEMs) and terminations (PLETs), pipeline sleepers, and riser bases are frequently supported with small shallow foundations. Dimmock et al. (2013) have studied the effect of adding short piles to the corners of the shallow foundation to improve the total capacity and reduce foundation footprints through numerical and physical modeling. The sliding bearing capacity of these foundations could also be significantly improved by placing short ribs or peripheral (and internal) foundation skirts. Typically rectangular foundations with lengths from $L=2$ to $10 \mathrm{~m}$ and width to length $(W / L)$ aspect ratios of 2:1 are used. The embedment (or skirt) depth $(d)$ to length $(L)$ ratios are up to $d / L=0.5$, but more typically 0.05-0.2 (Randolph et al. 2011). Although design codes (e.g. DNV, 1992; API, 2000 and ISO, 2003) have general comments on considering the effect of fishing gear and over-trawling in the design which are the sources of combined horizontal and torsional loading, they do not offer explicit methods for taking the in-plane horizontal loads and torsion into account for foundation design purposes.

This study considers an eccentrically loaded shallow foundation (Figure 1 ) of length $L$, width $W$ and embedment depth $d$. In this paper $L$ will be taken as the shorter dimension and will be aligned with the $x$-axis. In general, virtually any eccentricity angle $\psi$ or load angle $\gamma$ may oc- 
cur. This paper will first establish a number of baseline solutions for special eccentric/non-

eccentric loading cases where $\psi=0$ or $90^{\circ}$ and $\gamma=0^{\circ}$, for which relatively simple analytical or semi-analytical solutions may be derived from limit equilibrium or virtual work principles. For general conditions of eccentricity and load orientation, more powerful methods are required. Toward this end, the plastic limit analysis (PLA) proposed by Murff et al. (2010) is adopted. The accuracy of the baseline solutions and PLA approach are evaluated through comparisons to three-dimensional finite element solutions.

\section{BACKGROUND}

Murff and Miller (1977) used upper bound limit analysis to study the bearing capacity of multiple-footing offshore structures where they also studied the effect of torsion on collapse loads of foundations. Using limit equilibrium analysis Finnie and Morgan (2004) showed that the sliding resistance of the surface foundation could be decreased significantly if combined with torsion moments. They developed a normalized failure surface based on the following interaction equation:

$$
f(H, T)=\left[\left(\frac{H}{H_{\max }}\right)^{n}+\left(\frac{T}{T_{\max }}\right)^{k}\right]-1=0
$$

Where $H$ and $T$ are horizontal and torsion loads and $H_{\max }$ and $T_{\max }$ are the ultimate values. The interaction powers of $n$ and $k$ for surface foundations are recommended as $n=k=1.75$ for square and ranges of $n=1-2$ and $k=2-2.5$ for rectangular foundations. However, they did not conduct a rigorous analysis to validate their recommendations. They also presented some equations to estimate the ultimate torsional bearing capacity of surface foundation.

Yun et al. (2009) conducted three dimensional finite element (3D-FE) analysis to more rigorously study the interaction of vertical, horizontal, and torsion loads ( $V-H-T)$ acting on surface circular, square, and rectangular footings. The 3D-FE results of ultimate horizontal and tor- 
sional bearing capacity overestimated Finnie and Morgan (2004) limit equilibrium values by $4 \%$ and $11 \%$ respectively. They also developed normalized failure envelopes $\left(H / H_{\max }{ }^{-}\right.$ $\left.T / T_{\max }\right)$ for square and rectangular foundations of various aspect ratios.

Murff et al. (2010) developed an upper bound plastic limit analysis (PLA) formulation for shallow embedded square and rectangular foundations under combination of co-planar sliding and torsion. Figure 2 shows a schematic of the sliding-torsion failure mechanism. The mechanism comprises a rigid block DEFG of depth $d$ rotating about a vertical axis passing through the center of footing rotation $\left(x_{o}, y_{o}\right)$. The motion of the block results in formation of triangular wedges along each side of the foundation. Depending on the position of rotation center $\left(x_{o}\right.$ , $y_{0}$ ), these wedges undergo passive or active failure as indicated by letters "p" and "a”. Figure 2(b) shows a section view (CC') of the rigid block and wedges of $w_{\mathrm{i}}$. Examination of Section CC' shows that energy dissipation will occur at the discontinuities bounding the wedges (e.g., slip planes $\mathrm{A}_{1} \mathrm{~B}_{1}$ and $\mathrm{B}_{1} \mathrm{C}_{1}$ ) and within a zone of continuous deformation (e.g., volume $\left.\mathrm{A}_{1} \mathrm{~B}_{1} \mathrm{C}_{1}\right)$. Energy dissipation also occurs in the vertical planes at both ends of each wedge due to velocity jump from the wedge to the surrounding rigid soil. Finally, energy dissipation occurs due to slip at the base of the rigid block DEFG relative to the underlying rigid soil. Owing to the inclusion of both active and passive zones in the wedge the work rate done by the unit weight of the soil is zero. The equations for evaluation of the energy dissipation $D_{i}$ from the sources described above in terms of a virtual rotation rate $\dot{\beta}$ given by Murff et al. (2010) are contained in Appendix A.

The magnitude of force $H$ passing through load application point $\left(x_{f}, y_{f}\right)$ is calculated by 96 equating external work to internal energy dissipation:

$$
H=\frac{\sum_{i=1}^{5} \dot{D}_{i}}{\left[\left(x_{f}-x_{0}\right) \sin \theta+\left(y_{f}-y_{0}\right) \cos \theta\right] \dot{\beta}}
$$


97 where $\sum \dot{D}_{i}$ is the total rate of energy dissipation, $\theta$ is the angle between line of action of the external force and $x$-axis. A least upper bound is obtained by minimizing $H$ with respect to the rotation center coordinates $\left(x_{0}, y_{0}\right)$, the width of the deforming wedges $\left(w_{1}, w_{2}, w_{3}\right.$, and $w_{4}$ ), and the depth of the failure plane $(d)$ as optimization variables. Absent an underlying weak soil layer, the optimal depth of the failure plane generally coincides with the skirt penetration depth, in which case $d$ need not be considered as an optimization variable.

\section{BASELINE SOLUTIONS}

105 For limiting load conditions of eccentricity angle $\psi=0$ or $90^{\circ}$ and load angle $\gamma=0$ or $90^{\circ}$, a number of analytical and semi-analytical solutions are possible which can provide convenient reference solutions for evaluating PLA and FEA solutions.

\section{Pure sliding and rotation}

109 For pure sliding in a homogeneous soil with an adhesion factor $\alpha$ at the soil-skirt interface

110 and foundation embedment $d$, the bearing factors for sliding in the $x$ and $y$ directions derived

111 from the assumed stress distributions in Figure 3 and the failure mechanism in Figure 2(c)

112 are:

$$
N_{\text {x max }}=\frac{H_{x \max }}{s_{u} L W}=\alpha_{\text {base }}+2\left[(2+0.8 \alpha)+\alpha \frac{L}{W}\right] \frac{d}{L}
$$

$$
N_{\text {ymax }}=\frac{H_{\text {ymax }}}{s_{u} L W}=\alpha_{\text {base }}+2\left[(2+0.8 \alpha) \frac{L}{W}+\alpha\right] \frac{d}{L}
$$

115 where $s_{u}$ is the soil undrained shear strength, $L$ and $W$ are the smaller and larger sides of the

116 foundation. A different adhesion factor $\alpha_{\text {base }}$ is introduced here to allow for the case of a

117 skirted foundation, in which case $\alpha_{\text {base }}=1$. Otherwise, it would equal the adhesion factor $\alpha$ 118 applicable to the sides of the foundation. 
119 The stress distribution normal to the foundation skirts in Figure (3) is equivalent to lateral 120 earth pressure which is obtained using the upper bound limit analysis for a Coulomb wedge

121 forming behind the foundation skirt. Lateral pressure (i.e. bearing factor) is equal to $2 s_{\mathrm{u}}$ and $1222.8 s_{\mathrm{u}}$ for adhesion factor, $\alpha$, equal to 0 and 1 , respectively. For intermediate $\alpha$, a linear inter-

123 polation between the two values resulted in lateral pressure of $(2+0.8 \alpha) \times s_{\mathrm{u}}$ (Figure (3)).

124 For the special case of pure torsion with zero embedment, $d=0$, the following closed form 125 solution (Murff et al., 2010) for maximum torsional capacity $T_{\max 0}$ (the subscript ' 0 ' referring 126 to zero embedment) provides a useful check for numerical calculations:

$$
\begin{aligned}
& N_{t \max 0}=T_{\max 0} / s_{u} W L^{2}= \\
& \frac{(W / L)^{2}}{6}\left\{\frac{\sin \theta_{o}}{2 \cos ^{2} \theta_{o}}+\frac{\ln \left[\tan \left(\pi / 4+\theta_{o} / 2\right)\right]}{2}\right\}+\frac{L / W}{6}\left\{\frac{\cos \theta_{o}}{2 \sin ^{2} \theta_{o}}-\frac{\ln \left[\tan \left(\theta_{o} / 2\right)\right]}{2}\right\}
\end{aligned}
$$

128 where $\theta_{o}=\tan ^{-1}(L / W)$.

129 A limit equilibrium analysis obtained by summing the torsion resistance on the edges of a rotating footing (Figure 3) assuming no interaction effects between bearing and tangential resistance will produce the following expression for the additional torsional resistance derived

132 from footing embedment:

$$
\Delta N_{t \max e}=C_{f}\left[(1+0.4 \alpha)\left(\frac{W}{L}+\frac{L}{W}\right)+2 \alpha\right] \frac{d}{L}
$$

134 A correction factor $C_{f}$ is included in Eq. 6 to emphasize that some adjustment for interaction 135 effects is needed. The total torsional resistance is the sum of Eqs 5 and $6, N_{\text {tmax } 0}+\Delta N_{\text {tmaxe }}$.

136 This closed-form expression provides a useful glimpse into the variables affecting torsional

137 capacity and, as will be seen, a simple calibration is possible to provide a match to finite ele138 ment solutions.

\section{Combined loading}


140 For the case no embedment $(d / L=0)$ and eccentricity and loading directions aligned with ei-

141 ther the major or minor axis of the footing, a virtual work analysis is possible as shown in

142 Figure 4. A horizontal load $H$ is applied at a distance $e$ from the center of the footing with an

143 associated motion about a center of rotation located a distance $\rho$ from the center. Equating ex-

144 ternal virtual work $\dot{W}$ to internal energy dissipation leads to:

$145 \quad H=\dot{D} /(\rho+e) \dot{\beta}$

146 where $\dot{\beta}$ is a virtual angular velocity. The rate of internal energy dissipation $\dot{D}$ is the soil re-

147 sistance times the local velocity integrated over the footing area:

$$
\dot{D}=s_{u} \dot{\beta} \int_{-W / 2}^{W / 2} \int_{-L / 2}^{L / 2} \sqrt{(\rho+x)^{2}+y^{2}} d x d y
$$

149 A least upper bound collapse load is obtained by minimizing $H$ with respect to $\rho$ and setting

150 to zero, which leads to:

$151 \quad H=\frac{1}{\dot{\beta}} \frac{\partial \dot{D}}{\partial \rho}$

152 For the case of zero foundation embedment the collapse load then becomes:

$153 \quad H=s_{u} \int_{-W / 2}^{W / 2} \int_{-L / 2}^{L / 2} \frac{\rho_{\text {opt }}+x}{\sqrt{\left(\rho_{\text {opt }}+x\right)^{2}+y^{2}}} d x d y$

154 where $\rho_{\text {opt }}$ is the distance to the optimal center of rotation corresponding to a least upper

155 bound. Evaluation of the integral yields the following closed-form expression for $H$ :

156

$$
\begin{aligned}
& H=s_{u}\left[a_{1}^{2} \ln \left|\frac{b_{1}+W / 2}{b_{1}-W / 2}\right|-a_{2}^{2} \ln \left|\frac{b_{2}+W / 2}{b_{2}-W / 2}\right|+W\left(b_{1}-b_{2}\right)\right] \\
& a_{1}=\rho_{\text {opt }}+L / 2 \\
& a_{2}=\rho_{\text {opt }}-L / 2 \\
& b_{1}=\sqrt{a_{1}^{2}+W^{2} / 4} \\
& b_{2}=\sqrt{a_{2}^{2}+W^{2} / 4}
\end{aligned}
$$


157 With $\dot{D}$ and $H$ evaluated from Eqs. 8 and 11, respectively, the eccentricity $e$ associated with 158 any arbitrarily selected $\rho$ is evaluated from Eq. 7. Analytical integration of Eq. 8 is unwieldy; 159 however, reduction to single integration is possible (Appendix B) to facilitate simple design 160 spreadsheet calculations. As this method explicitly relates eccentricity to the distance to the 161 optimal center of rotation, it requires no search or optimization procedure. Aside from the simple numerical integration required to evaluate the rate of energy dissipation, the method is formulated in terms of closed-form expressions. Thus, it can provide a simple robust tool for routine design calculations.

Performing the analysis for a sweep of $\rho$ values yields the predicted reduction in load capacity $\left(H / H_{\max }\right)$ versus eccentricity $e$ as shown in Figure 5, which shows the significant reduction in sliding resistance for loading in the $x$ and $y$ directions. Square footings, $W / L=1$, experience the greatest reductions in capacity $H$, with eccentricity as low as $e / L=0.1$ reducing load capacity by more than $5 \%$ and eccentricity $e / L=0.5$ (load application at the edge of the footing) reducing capacity by more than $40 \%$. With increasing aspect ratio $\mathrm{W} / \mathrm{L}$ the foundation becomes progressively more resistant to eccentric loading. The predictions also show that the reduction in capacity is always greatest for loading in the $x$ direction; i.e., for load orientations normal to the long axis of the foundation.

\section{FINITE ELEMENT ANALYSES}

A series of finite element analyses (FEA) were performed to provide a basis for evaluating the various analytical procedures described above. The commercial software ABAQUS (HKS, 2008) is used in this study.

\section{Geometry and material parameters}

180 Clay under undrained conditions is modeled as linear elastic perfectly plastic material, obeying a von Mises failure criterion and an associated flow law. Undrained shear strength in a 
simple shear mode, denoted by $s_{\mathrm{u}}$, is used in order to allow comparison with Tresca based an-

183 alytical solutions, since Von Mises criterion with the simple shear undrained strength pro-

184 vides a reasonable approximation of the Tresca criterion. Furthermore, Gourvenec et al.

185 (2006) showed a very close agreement of the vertical bearing capacity of the surface footing

186 for the Von Mises with simple shear strength and Tresca criteria. The Young's modulus of

187 the soil is taken as a multiple of undrained shear strength $E / s_{u}=500$. The ultimate capacity of

188 the foundation is not affected by the pre-failure elastic behavior of the soil (Chen and Liu

189 1990), so elastic behavior is introduced purely for the purpose of numerical implementation

190 of the finite element analysis. Poisson's ratio is taken as 0.49 to simulate no volume change

191 for undrained clay in a total stress analysis. The foundation is modeled as a rigid body with

192 Young's modulus $10^{10}$ times that of the soil. Strictly speaking, the solutions presented apply

193 to a solid block foundation, although the predictions can be reasonably applied to skirted

194 foundations provided no weak layers are encompassed by the skirts. No separation is assumed

195 is assumed to occur at the foundation-soil interface. The interaction between soil and founda-

196 tion is modeled using surface to surface contact pair in which footing outer surface is chosen

197 as a "master surface” and the soil surface in contact with the foundation as a "slave surface”.

\section{Finite element model}

199 Figure 6 shows an example 3D mesh and various zones of the model for a rectangular foun-

200 dation. The length $(L)$ and width $(W)$ of the footing are parallel to $x$ and $y$ coordinates respec-

201 tively. The model dimensions are $9 L \times 9 L \times 5 L$ and $9 L \times 12 L \times 5 L$ for the square and rectangular

202 foundations respectively. The 3D mesh consists of 8-node full- integration 3D hybrid first or-

203 der elements. The shallow foundation is simulated as square and rectangular $(W / L=2)$ shapes

204 with various embedment depths of $d=L / 40, L / 28, L / 20$, and $L / 14$. In order to create transitions in mesh density within the model the whole mesh is divided to 6 zones. The finite ele- 
transition of mesh density. This constraint ties active degree of freedom of the common surfaces along the transition interface and therefore the two surfaces at the transition zone do not necessarily have common nodes. The finest zone extending to $2 d$ around the foundation em-

210 bedment consists of two way bias grading of elements across the footing area as well as the

211 height of the footing skirt. The size of the elements under the footing is very small, less than

$2120.006 L$. The bias grading of elements also provides adequately fine mesh of soil with dimen-

213 sion of $0.0004 \mathrm{~L}$ to $0.0008 \mathrm{~L}$ in the vicinity of the sharp edges of the rigid foundation. The final

214 FE mesh was obtained after checking various mesh densities especially for the finest zone,

215 which has the main impact on the accuracy of the ultimate results as well as computational

216 expense of the numerical simulation.

\section{Boundary conditions and loading}

218 Full fixity in all directions is enforced at the base boundary condition, while lateral fixity is 219 enforced at the far-field vertical boundaries. The analysis is conducted using displacement control until ultimate sliding and torsional resistance is mobilized. This typically required translation in excess of $u / L=0.003$ and rotations greater than 0.02 radians as shown in Figures 7 and 8. All the FEA simulations are conducted for the foundation fully bonded to the soil.

In order to construct the interaction curve or yield locus for combined shear-torsion loading, two types of displacement control methods are used: the swipe test and the probe test. The swipe test was used by Tan (1990) during his centrifuge tests to determine the shape of failure envelope. The advantage of this approach is that yield locus is determined by one single analysis in two separate steps. In the swipe test the foundation is firstly displaced in the direction of the degree of freedom (DoF) under examination from zero to ultimate capacity until the collapse load in that direction is reached. In the second step, the displacement is imposed in the second DoF until the ultimate capacity in the new direction is fully developed. In the 
small strain finite element once the ultimate force is reached in the first step, there will be no more increase in the reaction force in that DoF, thus no further expansion in overall failure

234 locus of foundation as the footing movement progresses. The disadvantage of this method is that, due to the elasto-plastic yielding occurring within the failure locus, the swipe test tracks a load path marginally inside the actual overall foundation failure envelope (Bransby and Randolph 1998). In the probe test or fixed displacement method, a single point on the failure envelope is identified for every fixed ratio of the prescribed combined displacement. Therefore, the yield locus could be created by conducting a number of finite element analyses with different displacement ratios. The prescribed fixed displacement ratio gives rise to load path beginning from the origin with initial gradient based on elastic stiffness. As approaching failure envelope the gradient diminishes to follow the interaction curve until it stops where there is no further increase in the forces developed in each intended degree of freedom (Bransby and Randolph 1998). This method gives accurate failure envelope, but requires several tests.

\section{COMPARISON TO BASELINE AND PLA SOLUTIONS}

The finite element studies are now compared to the baseline and PLA solutions from two perspectives. Firstly, the size of the yield envelope is evaluated through comparisons of predicted capacities under pure translational and rotational loading, $H_{x \max }, H_{y \max }$, and $T_{\max }$. Secondly, the shape of the yield envelopes are evaluated through comparisons to a yield envelope in normalized space, $f\left(H_{x} / H_{x \max }, H_{y} / H_{y \max }, T / T_{\max }\right)=0$.

\section{Pure translation and rotation}

Figure 9 presents the computed bearing factors versus embedment depth. For both sliding and torsion, maximum capacity increases linearly with increasing embedment depth $d / L$ as predicted from the LE solutions in Eqs. 3-6. The FEA sliding bearing factors are in excellent 
sional resistance bearing factors $N_{t}$ match Eq. 5 to within $0.1 \%$. As embedment increases, the uncorrected $\left(C_{f}=1\right)$ LE solutions are seen to increasingly exceed the FE solutions, by up to $7.5 \%$ for $W / L=1, d / L=1 / 14$. It can be shown that an interaction factor $C_{f}=0.87$ in Eq. 6 will bring the limit equilibrium predictions into close agreement with finite element solutions for both the square and rectangular footings.

For the case of zero embedment, $d / L=0$; the FEA results are in essentially very close agreement with the PLA. As embedment increases the PLA tends to over-estimate capacity somewhat, which is in accordance with expectations for an upper bound solution. For translational resistance, $N_{\text {sxmax }}$ and $N_{\text {symax }}$, the difference between FEA and PLA is, with one exception, less than $1 \%$. For the case of pure torsion the difference between FEA and PLA become more severe with increasing embedment depth, and can exceed $6 \%$ for $d / L=1 / 14$. The largest differences between FEA and PLA occur for the case of a square foundation. Overall, the situation for the PLA is similar to that of the LE analysis; PLA estimates of pure translational load capacity agree very well with FEA solutions, but the PLA tends to overestimate the increase in torsional capacity due to foundation embedment. Close examination indicates that the PLA overestimates the additional torsional resistance derived from embedment by about $10 \%$.

273 Figure 10 shows contours of plastic displacements for the cases of pure sliding and pure rotation. Figure 10a clearly shows the wedge formation at the ends of the foundation that is assumed in the PLA analysis. Figure 10b shows a simple pattern at the base of the foundation comprising circular contours about the center of rotation. At the edges of the foundation more complex displacement patterns develop as the wedges rotate and distort. Interestingly negligible plastic deformation is observed in the soil elements next to the corners of the foundation. This is consistent with Murff et al. (2010) PLA mechanism depicted in Figure 2, which assumes rigid soil at the corners of the foundation. 


\section{Combined loading}

282

283

284

285

286

287

288

289

290

291

292

293

294

295

296

297

298

299

300

301

302

303

304

305

Figures 11 and 12 show example FEA interaction diagrams for a rectangular $(W / L=2)$ foundation with embedment $d / L=1 / 14$ under combined loading. Figure 11 shows the shearxtorsion interaction, while Figure 12 shows the $x$-sliding and $y$-sliding interaction. These illustrate the trend of swipe tests tending to give an apparent yield locus marginally inside the actual locus. Accordingly, yield loci taken from the probe approach are adopted as more reliable.

Figures 13- 15 compare the FEA shear-torsion interaction predictions to PLA solutions and to the zero embedment $(d / L=0)$ virtual work analysis Eqs. 7-11. The figures present the computed load capacities in normalized form, $H / H_{\max }$ and $T / T_{\max }$ for the case of a square footing (Figure 13) and the $x$ and $y$ sliding for a rectangular footing (Figures 14 and 15, respectively.) The FEA predictions show the shape of the yield envelopes to be insensitive to embedment depth, with all predictions for the embedment range $d / L=1 / 28$ to $1 / 14$ essentially lying on a single unique curve. The yield envelopes from the PLA method are in general agreement with the FEA solutions, although the PLA is slightly unconservative for the square footing (Figure 13). Remarkably, the virtual work solution for zero embedment also provides a realistic portrayal of the yield envelope for non-zero embedments, with some tendency for being on the conservative side, especially for the $W / L=2$ footing loaded in the $y$ direction (Figure 15).

Figure 16 shows the yield envelopes for the case of non-eccentric loading for varying load directions $\gamma$. Again the FEA solutions indicate that embedment $d / L$ has minimal influence on the shape of the envelope. The PLA predictions agree well with the FEA solutions, albeit with a slight tendency for being on the unconservative side. For the case of zero embedment, the yield envelope obtained from the PLA approach (Eqs. 10 and 11) will be circular. Plastic Limit Analysis (PLA) approach produces the circular yield envelope for the foundation with zero embedment. This concept is also easy to explain theoretically: for foundation with area 
of base $=A$, embedment $=0.0$, and soil undrained shear strength $=s_{u}$ the sliding resistance

307 will be constant and equal to $H_{\max }=H_{x, \max }=H_{y, \max }=A s_{u}$, regardless of the direction of slid-

308 ing. This value actually represents the radius of the circular yield curve in the $H_{x}-H_{y}$ space as

309 shown in Figure 16. The components of sliding resistance in $x$ and $y$ directions are $H_{x}=$ $H_{\max } \times \cos \eta$ and $H_{y}=H_{\max } \times \sin \eta$ for the angle of foundation sliding with respect to $x$ axis equal to $\eta$. This circular interaction relationship is correct for foundation with any geometric base shape when embedment is zero. Figure 16 indicates that embedment of the footing will induce a small but noticeable departure from a circular envelope. This is also the case for square footing. A simplifying assumption of a circular $H_{x} / H_{x, \max }-H_{y} / H_{y, \max }$ yield envelope should therefore produce slightly conservative yet realistic predictions. Thus, Eq. 1 could be rewritten as below:

$$
f\left(H_{x}, H_{y}, T\right)=\left\{\left[\left(\frac{H_{x}}{H_{x, \max }}\right)^{p}+\left(\frac{H_{y}}{H_{y, \max }}\right)^{q}\right]^{r}+\left(\frac{T}{T_{\max }}\right)^{k}\right\}-1=0
$$

where for the assumed simplified circular relationship between $H_{x}$ and $H_{y}$ we have $p=q=2$.

The interaction relationship in Eq. 12 represents the ellipsoidal surface in the $H_{x}-H_{y}-T$ space.

\section{LOAD CAPACITY REDUCTION DUE TO ECCENTRICITY}

321 An alternative approach to view and evaluate the combined loading effects is to relate the load capacity to eccentricity as shown in Figures $17-20$, for $W / L=1,2$, 4, and 6. Figures 1820 also include the effects of eccentricity angle, showing the predictions for $\psi=0$ and $90^{\circ}$

324 when $\gamma=0^{\circ}$ (sliding in $y$ and $x$ directions with various eccentricity). In these figures the load capacity is expressed in terms of bearing factor form $\left(N=H / s_{u} L W\right)$ so the comparisons reflect the differences in the computation of both the magnitude of resistance as well as the interaction effects. Also in Figures 17 and 18, the FEA results are shown, which indicate gener- 
ally good agreement between the FEA and PLA solutions, although the PLA predictions are somewhat high for the square foundation.

330 The insensitivity of the shape of the yield locus to variations in embedment depth $d / L$ (Figures 13-15) introduce the possibility of developing a simplified approach to evaluating sliding-torsional capacity using the simple baseline solutions presented earlier. For instance, the relationships for translational load capacity (Eqs. 3 and 4), which proved to be fairly accurate, can be used in conjunction with the virtual work equations for zero embedment (Eqs. 7-11, Figure 5) to make first order predictions of load capacity. As an example, if the maximum horizontal load capacity values from Eqs. 3 and 4 are used with the corresponding capacity reduction curves for $x$ and $y$ directional loading from Figure 5, simple estimates of horizontal sliding resistance versus eccentricity are possible as shown in Figures 17-20 for aspect ratios $W / L=1$ to 6 . Comparisons to FEA and PLA solutions indicate that the simplified approach provides realistic, albeit conservative, estimates of load capacity reduction due to eccentricity.

341 Although the PLA can be performed easily with minimal computation time, also performing the simplified analysis is advisable. The reason for this is that the PLA performs an optimization analysis to search for a least upper bound collapse load $H$. The possibility always exists that the search may identify a local minimum that is greater than the absolute minimum. The simplified analysis provides a useful benchmark that can aid in judging if the absolute lower bound has been found.

347 Up to this point the analyses under consideration have been restricted to conditions for which 348 the load angle $\gamma=0$. A question now arises as to how to deal with cases of arbitrary load an349 gles. The issue is clearly significant, since increasing $\gamma$ will decrease the torsional loading and 350 for $\gamma=90^{\circ}$ the torsion vanishes. The virtual work approach cannot handle the cases of $\psi \neq 0$ or $90^{\circ}$ and $\gamma \neq 0$ or $90^{\circ}$. However, response of shallow foundation subjected to any combina- 
tion of coplanar translation and torsion could be evaluated through PLA solution and appropriate factors of $p, q, r$, and $s$ in Eq. 12 could be determined to define the ellipsoid interaction surface.

\section{CONCLUSIONS}

This study evaluates the ultimate sliding resistance of square and rectangular shallow embedded foundations under purely horizontal eccentric loads. 3D-FE simulations are performed to evaluate a number of simple relationships developed for special conditions of loading as well as an upper bound plastic limit analysis (PLA) developed by Murff et al. (2010) for general conditions of shear-torsional loading. The study indicates the following:

1. Eccentricity of lateral loading begins to significantly affect ultimate sliding resistance of square footings ( $>5 \%$ reduction in capacity) at eccentricity levels $e / L$ greater than about 0.1 (Figure 5). For perspective, an eccentricity $e / L=0.5-$ a load applied at the edge of the footing - reduces capacity by more than $40 \%$.

2. Foundation performance with respect to eccentric loading improves with increasing aspect ratio $W / L$ (Figure 5). The load capacity reduction due to eccentricity is always greater for loading in the direction normal to the long axis of the foundation.

3. The limit equilibrium equations for translational load capacity, Eqs. 3 and 4, give reliable predictions for pure translational loading (Figure 9). Eq. 5, which predicts pure torsional load capacity for the case of no footing embedment $d / L=0$, is also accurate. The limit equilibrium solution for the increase in torsional capacity for $d / L>0$ (Eq. 6) can provide reasonably accurate solutions when used in conjunction with a correction factor $C_{f}=0.87$.

4. Predictions from the PLA method of Murff et al. (2010) generally agree well with FEA predictions (Figures 13-15 and 17-18). The method over-predicts the increase in 
pure torsional resistance associated with increased embedment $d / L$; however, this appears to have minimal effect on the quality of the predictions for eccentricity levels likely to be encountered in practice.

5. The general insensitivity of the shape of the shear-torsion yield envelope to embedment depth $d / L$ (Figures 13-15) permits a simplified analysis in which analytical expressions for translational resistance (Eqs. 3 and 4) are used in conjunction with a virtual work analysis for the case of zero embedment (Eqs. 7-11) to predict the reduction in load capacity due to eccentricity. The method is fairly robust, as it requires no search for a least upper bound. Predictions from the simplified method provide reasonable conservative estimates of the reduction in sliding resistance due to eccentricity.

6. The effect of eccentricity angle $\psi$ increases with increasing aspect ratio $W / L$ (Figures 17-20). The simplified analysis does not accurately capture the effects of $\psi$ at large $W / L$ and large eccentricity for sliding in the $y$ direction (parallel to the long axis of the foundation), where it can be overly conservative. 


\section{REFERENCES}

API RP 2A, (2000). Recommended Practice for Planning, Designing and Constructing

Fixed Offshore Platforms-Working Stresses Design (21 $1^{\text {st }}$ Edition), American Petroleum Institute, Washington.

Bransby, M. F., and Randolph, M. F. (1998). “Combined loading of skirted foundations.”

Chen, W. F., and Liu, X. L. (1990). Limit analysis and soil plasticity. Elsevier Publishing Co., Amsterdam, The Netherlands.

400

Dimmock, P., Clukey, E., Randolph, M., Murff, D., and Gaudin, C. (2013). "Hybrid Sub401 sea Foundations for Subsea Equipment.” J. Geotech. Geoenviron. Eng., 139(12), 2182-2192. DNV, (1992). Foundations: Classification notes No. 30.4, Det Norske Veritas, Oslo. Int. Offshore and Polar Engineering Conference (ISOPE), Toulon, France. the Offshore Technology Conference (OTC), Houston.

Gourvenec, S.M., Randolph, M. F., and Kingsnorth, O. (2006). "Undrained Bearing Capacity of Square and Rectangular Footings.” Int. J. Geomech., 6(3), 147-157.

HKS Inc. (2008). ABAQUS Version 6.6 User's Manual. Hibbitt, Karlson and Sorensen, Inc, Pawtucket, Rhode Island.

ISO (2003). ISO 19901-4:2003(E): Petroleum and Natural Gas Industries-Specific Requirements for Offshore Structures, Part 4: Geotechnical and Foundation Design, ISO, Geneva.

Murff, J.D., and Miller, T.W. (1977). "Stability of offshore gravity structure foundations using the upper bound method.” Proc. of the Offshore Technology Conference (OTC), Houston, U.S.A., Paper OTC 2896. 
417 Murff, J.D., Aubeny, C.P., Yang, M. (2010). The effect of torsion on the sliding resistance 418 of rectangular foundations.” Proc. $2^{\text {nd }}$ Int. Symp. on Frontiers in Offshore Geotechnics 419 (ISFOG), Perth, Australia, 439-444.

420 Randolph, M.F., Gaudin, Ch., Gourvenec, S.M., White, D.J., Boylan, N., and Cassidy, 421 M.J. (2011) "Recent advances in offshore geotechnics for deep water oil and gas develop422 ments.” Ocean Engineering, 38(7), 818-834.

423 Tan, F. (1990). “Centrifuge and theoretical modelling of conical footings on sand.” Ph.D. 424 thesis, The University of Cambridge, Cambridge, U.K.

425 Yun, G.J., Maconochie, A., Oliphant, J., Bransby, M.F., (2009) “Undrained capacity of 426 surface footings subjected to combined V-H-T loading." Proc. $19^{\text {th }}$ Int. Offshore and Polar 427 Engineering Conference (ISOPE), Osaka, Japan.

428 
Appendix A. Energy Dissipation Rate Terms for PLA by Murff et al. (2010)

\begin{tabular}{|c|c|c|}
\hline Mechanism & Location & Equation \\
\hline \multirow{2}{*}{$\begin{array}{l}\text { Shear deformation } \\
\text { within wedges }\end{array}$} & Sides 1 and 3 & $W w_{1} \dot{\beta} \sqrt{1+\left(d / w_{1}\right)^{2}} \int_{z=0}^{z=d} s_{u}(z)(1-z / d) d z$ \\
\hline & Sides 2 and 4 & $L w_{2} \dot{\beta} \sqrt{1+\left(d / w_{2}\right)^{2}} \int_{z=0}^{z=d} s_{u}(z)(1-z / d) d z$ \\
\hline \multirow{2}{*}{$\begin{array}{l}\text { Slip between rigid } \\
\text { block DFEG and } \\
\text { vertical faces of } \\
\text { wedges }\end{array}$} & Sides 1 and 3 & $\alpha \dot{\beta} \int_{z=0}^{z=d} \int_{y=-W / 2}^{y=W / 2}\left[\left(\frac{L}{2}-x_{o}\right)^{2}+\left(\frac{d}{w_{1}}\right)^{2}\left(y-y_{o}\right)^{2}\right]^{\frac{1}{2}} s_{u}(z) d y d z$ \\
\hline & Sides 2 and 4 & $\alpha \dot{\beta} \int_{z=0}^{z=d} \int_{x=-L / 2}^{x=L / 2}\left[\left(\frac{W}{2}-y_{o}\right)^{2}+\left(\frac{d}{w_{2}}\right)^{2}\left(x-x_{o}\right)^{2}\right]^{\frac{1}{2}} s_{u}(z) d x d z$ \\
\hline \multirow{2}{*}{$\begin{array}{l}\text { Slip along the } \\
\text { wedge ramps }\end{array}$} & Sides 1 and 3 & $\dot{\beta} \sqrt{\left(d / w_{1}\right)^{2}+1} \sqrt{\left(w_{1} / d\right)^{2}+1} \int_{z=0}^{z=d} \int_{y=-W / 2}^{y=W / 2}\left|y-y_{o}\right| s_{u}(z) d y d z$ \\
\hline & Sides 2 and 4 & $\dot{\beta} \sqrt{\left(d / w_{2}\right)^{2}+1} \sqrt{\left(w_{2} / d\right)^{2}+1} \int_{z=0}^{z=d} \int_{x=-L / 2}^{x=L / 2}\left|x-x_{o}\right| s_{u}(z) d x d z$ \\
\hline \multirow{2}{*}{$\begin{array}{l}\text { Slip at the two } \\
\text { ends of each } \\
\text { wedge }\end{array}$} & Sides 1 and 3 & $w_{1} \dot{\beta}\left|W / 2-y_{o}\right| \sqrt{\left(d / w_{1}\right)^{2}+1} \int_{z=0}^{z=d}(1-z / d) s_{u}(z) d z$ \\
\hline & Sides 2 and 4 & $w_{2} \dot{\beta}\left|L / 2-x_{o}\right| \sqrt{\left(d / w_{2}\right)^{2}+1} \int_{z=0}^{L=a}(1-z / d) s_{u}(z) d z$ \\
\hline $\begin{array}{l}\text { Slip at the base of } \\
\text { rigid block DEFG }\end{array}$ & Base & $\dot{\beta} \int_{y=-W / 2}^{y=W / 2} \int_{x=-L / 2}^{x=L / 2} s_{u}(d) \sqrt{\left(x-x_{o}\right)^{2}+\left(y-y_{o}\right)^{2}} d x d y$ \\
\hline
\end{tabular}

Notes:

$w_{1}, w_{2}, w_{3}$, and $w_{4}$ : width of the failure wedges in soil

$\left(x_{0}, y_{0}\right)$ : coordinates of the center of rotation

$\left(x_{\mathrm{f}}, y_{\mathrm{f}}\right)$ : coordinates of the application point for the external force $H$

$\theta$ : inclination angle of the external force $H$ with respect to $x$ axis

$d$ : depth of the failure/sliding plane (assumed to be equal to embedment depth of the shallow foundation)

$z$ : depth below seabed

$\alpha$ : adhesion factor

$\dot{\beta}$ : virtual rate of rotation 
442 Appendix B. Integration of Dissipation Rate for $\boldsymbol{d}=\mathbf{0}$ analysis

443 Analytical evaluation of the inner integral in Eq. 8 is possible to permit a single numerical in-

444 tegration. The resulting expression is:

$\dot{D}=\frac{1}{2} s_{u} \dot{\beta} \int_{-W / 2}^{W / 2}\left[\left(a_{1} c_{1}-a_{2} c_{2}\right)+y^{2} \ln \left|\frac{a_{1}+c_{1}}{a_{2}+c_{2}}\right|\right] d y$

$a_{1}=\rho_{\text {opt }}+L / 2$

$445 \quad a_{2}=\rho_{\text {opt }}-L / 2$

$c_{1}=\sqrt{a_{1}^{2}+y^{2}}$

$c_{2}=\sqrt{a_{2}^{2}+y^{2}}$

446 Eq. B1 can be integrated between the limits $-W / 2$ to $W / 2$ using classical numerical integration 447 formulas. 


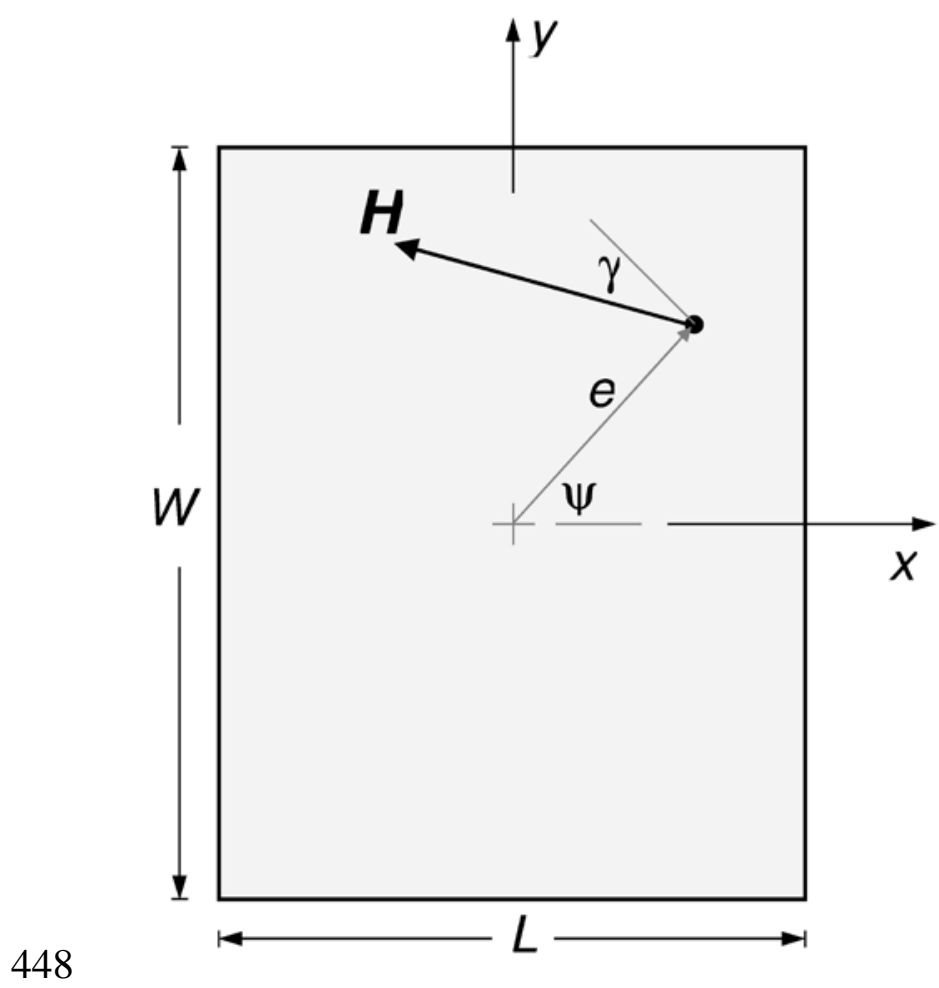

449 Figure 1. Schematic of eccentrically loaded foundation

450 


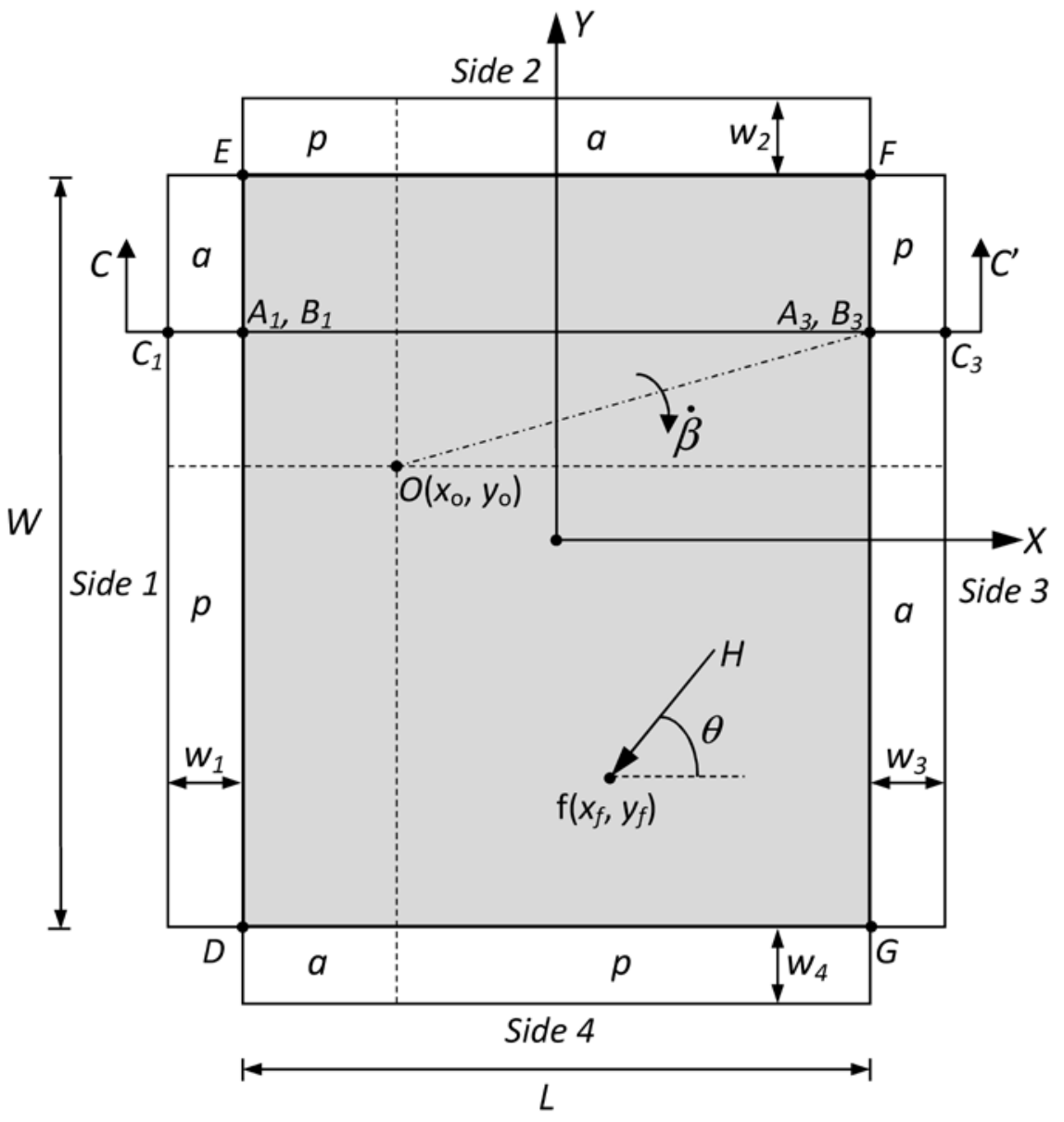

(a) Plan view of the mechanism

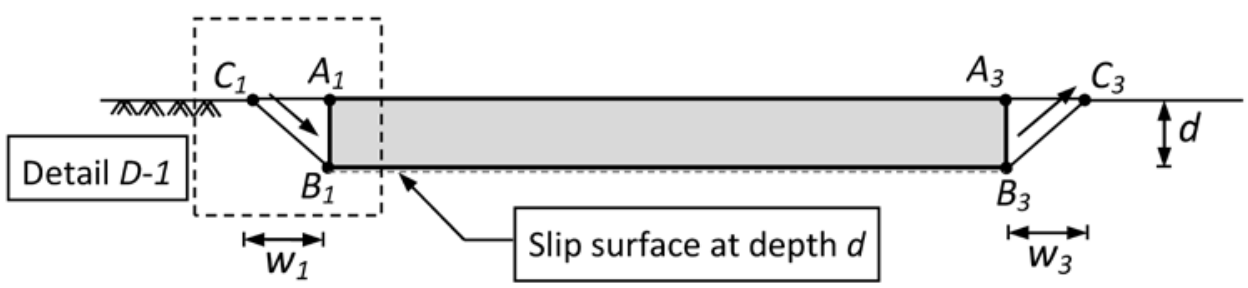

(b) Elevation view of section $C C^{\prime}$
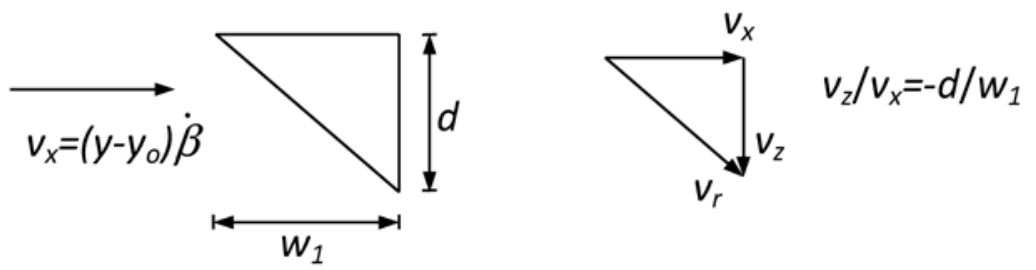

(c) D-1: Elevation view of active wedge $A_{1} B_{1} C_{1}$

452 Figure 2. Assumed collapse mechanism in PLA analysis 

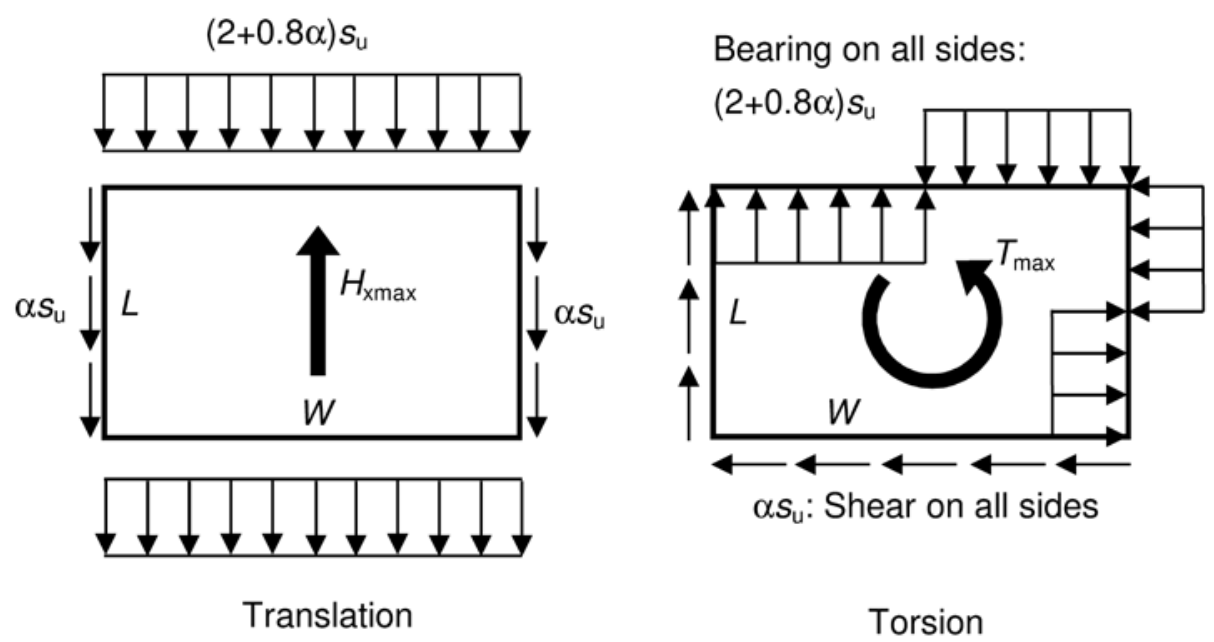

454

455 Figure 3. Assumptions for limit equilibrium equations 


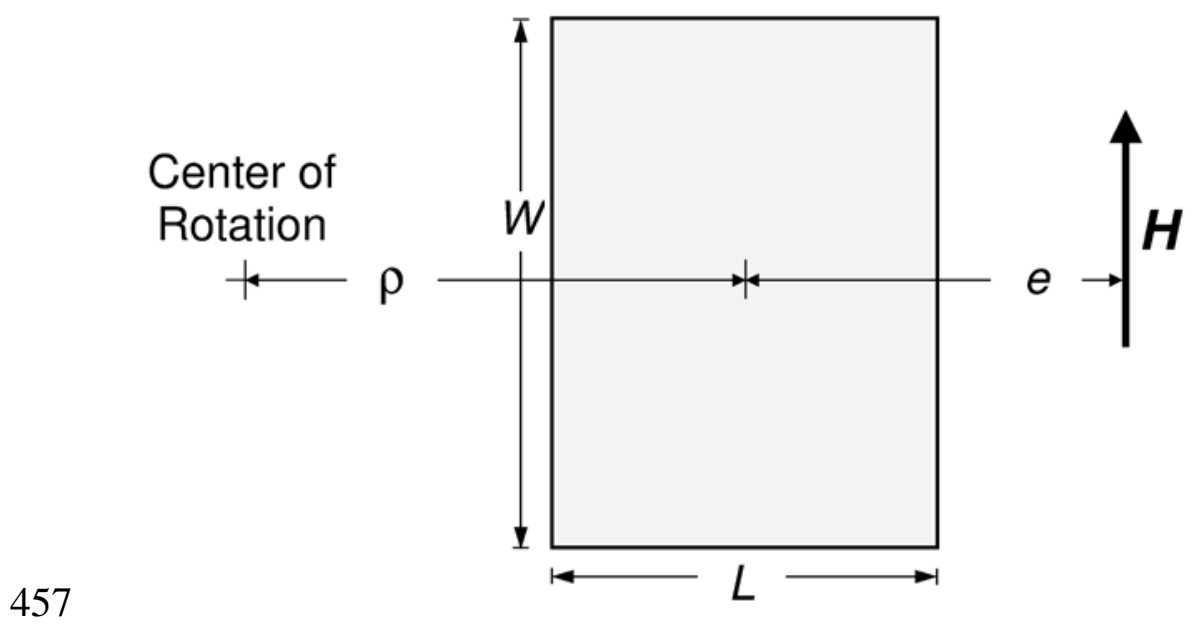

458 Figure 4. Model for virtual work analysis of shear-torsion

459 


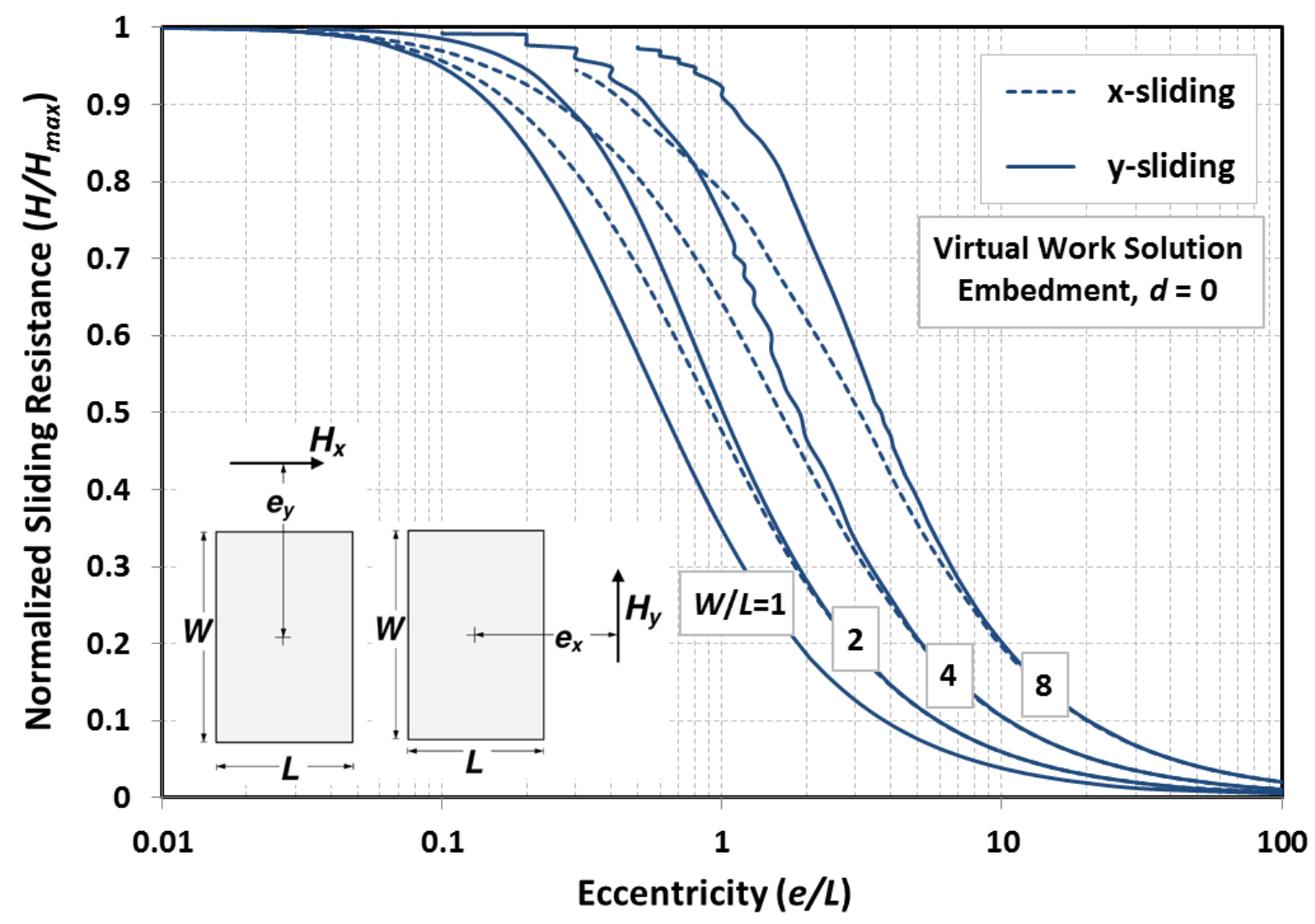

460

461 Figure 5. Horizontal capacity reduction predicted from virtual work analysis for surface foun462 dation $(d=0)$ 


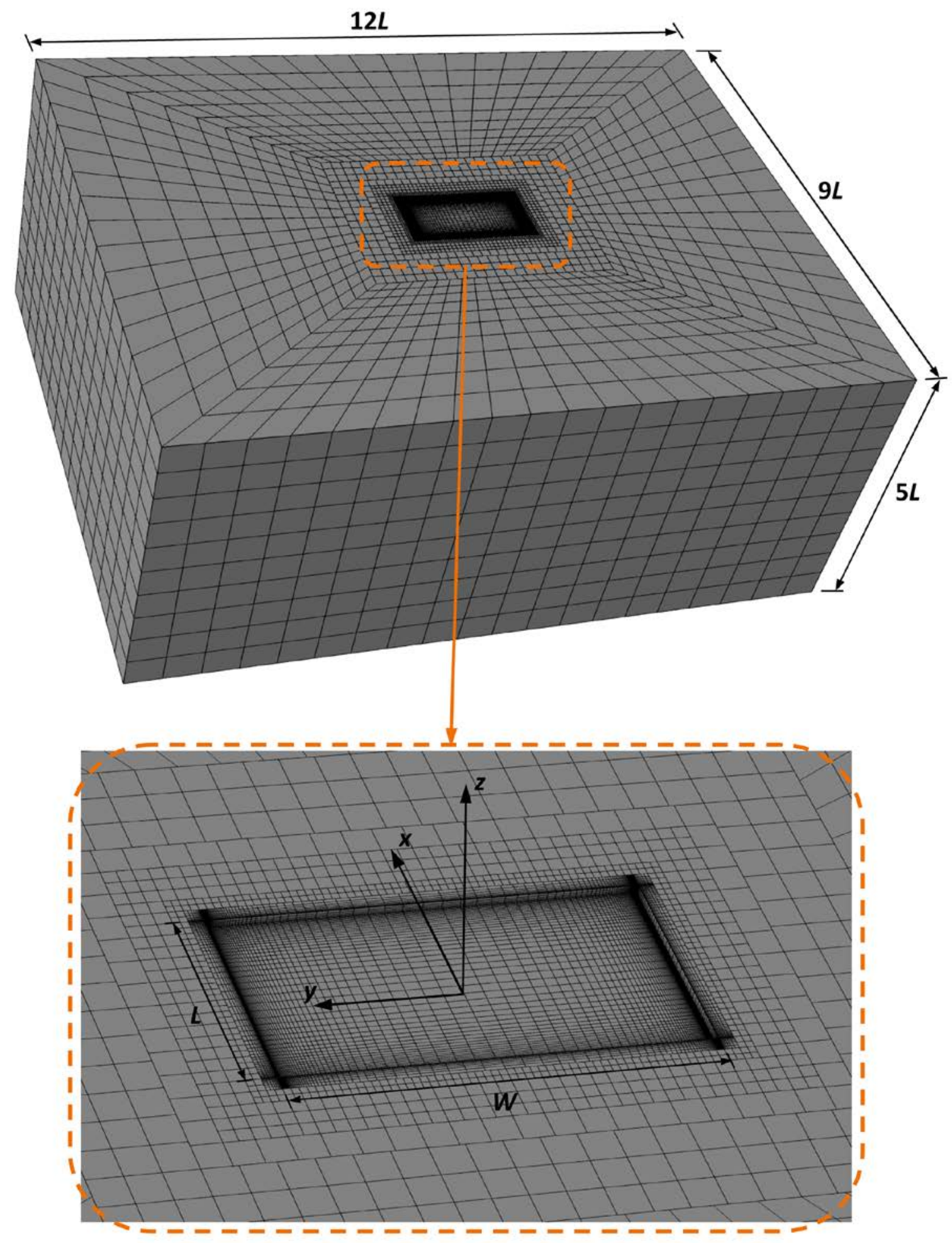

465 Figure 6. Finite element model 


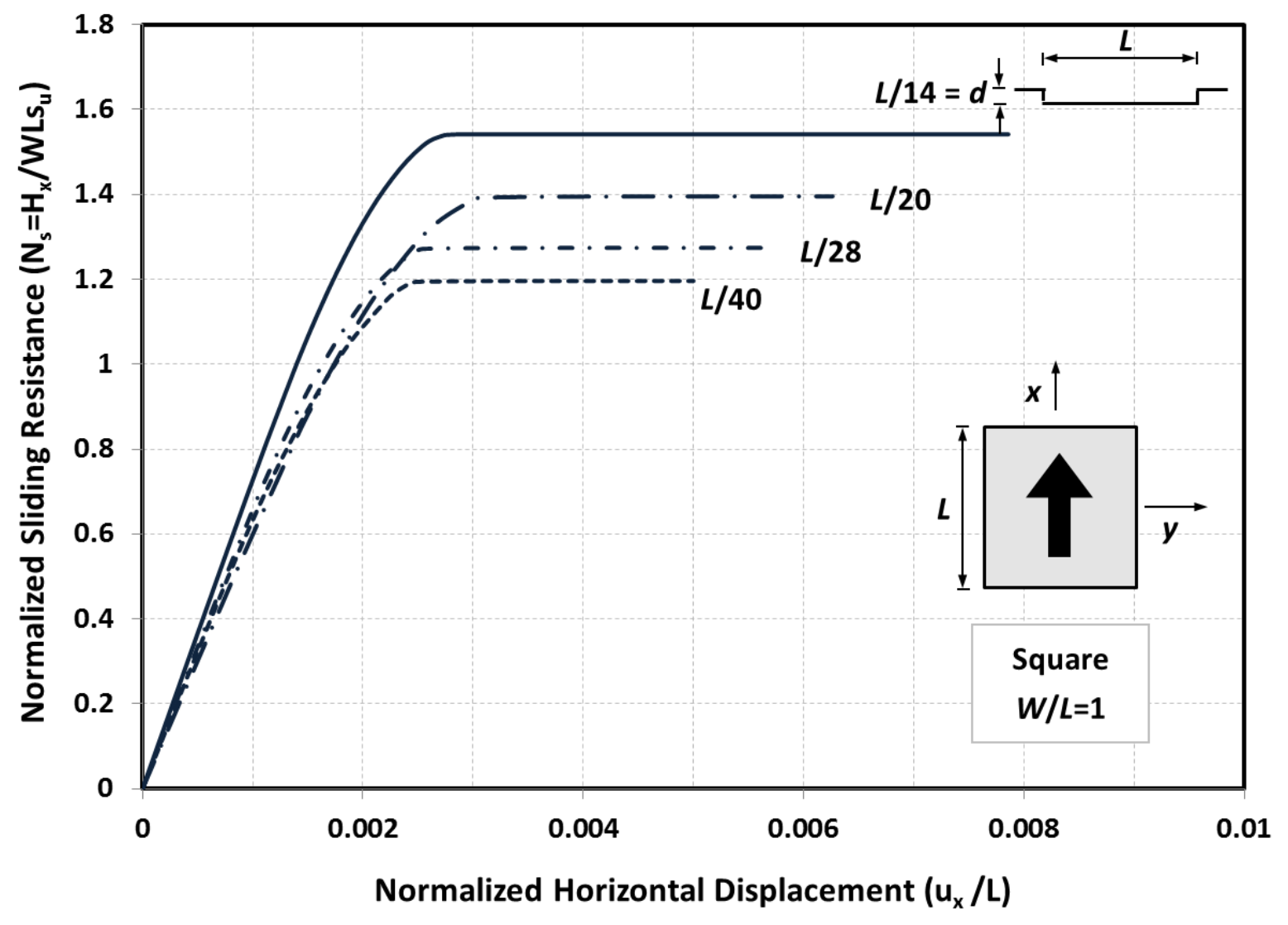

467

468 Figure 7. Typical load-displacement curve from FEA 


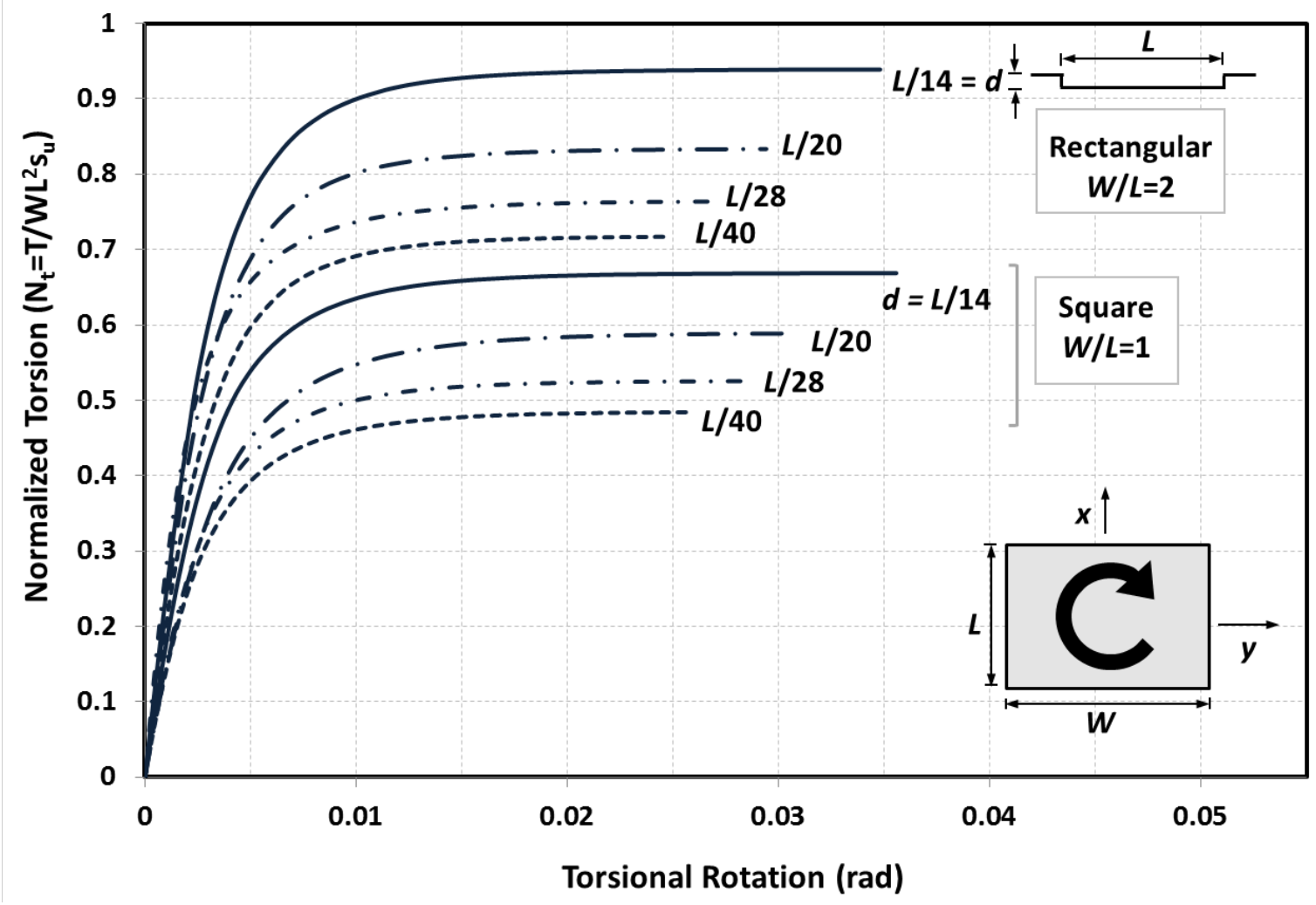

470

Figure 8. Typical torsion-rotation curve from FEA

472 


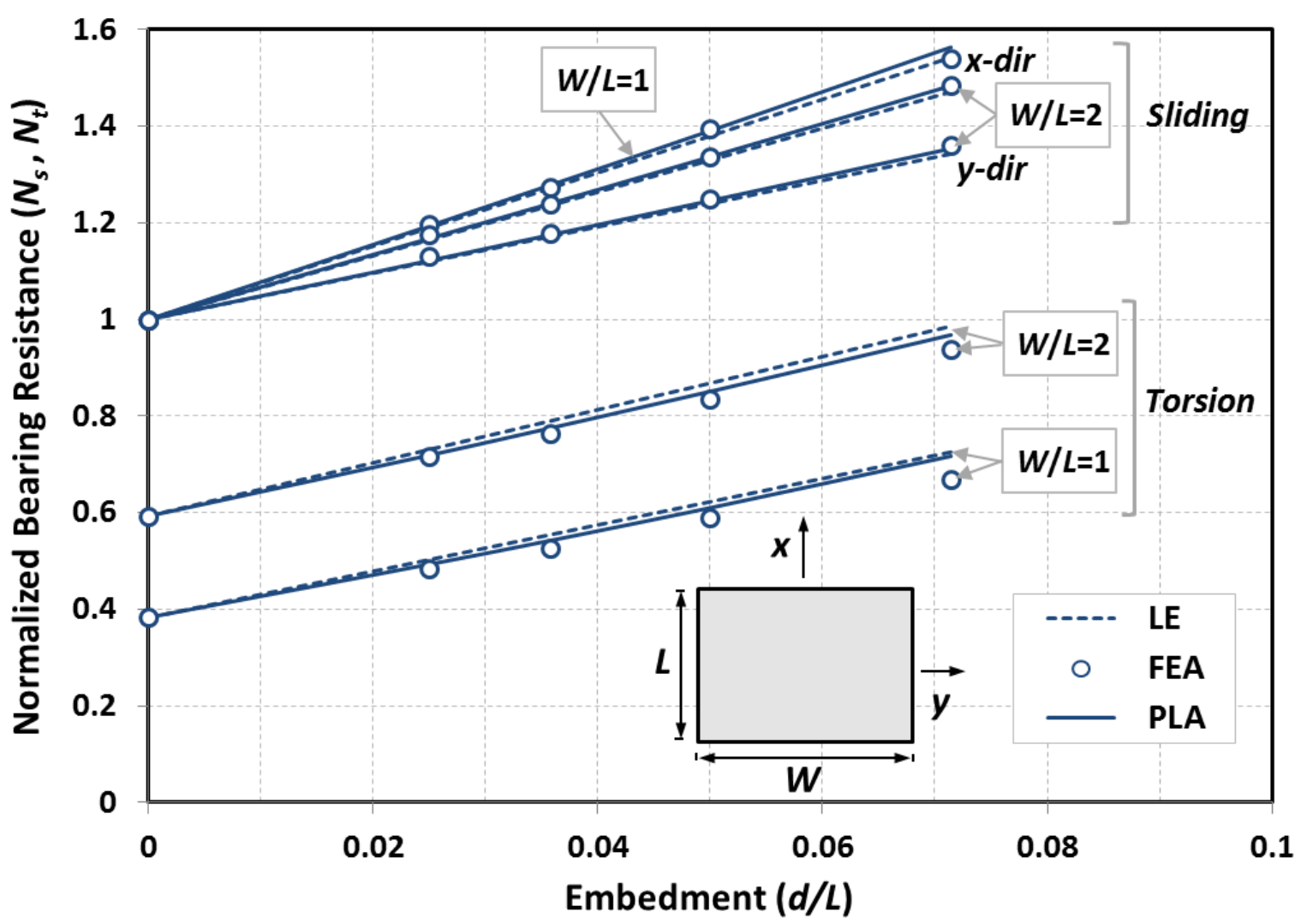

473

474 Figure 9. Normalized bearing capacity for pure translation and torsion 


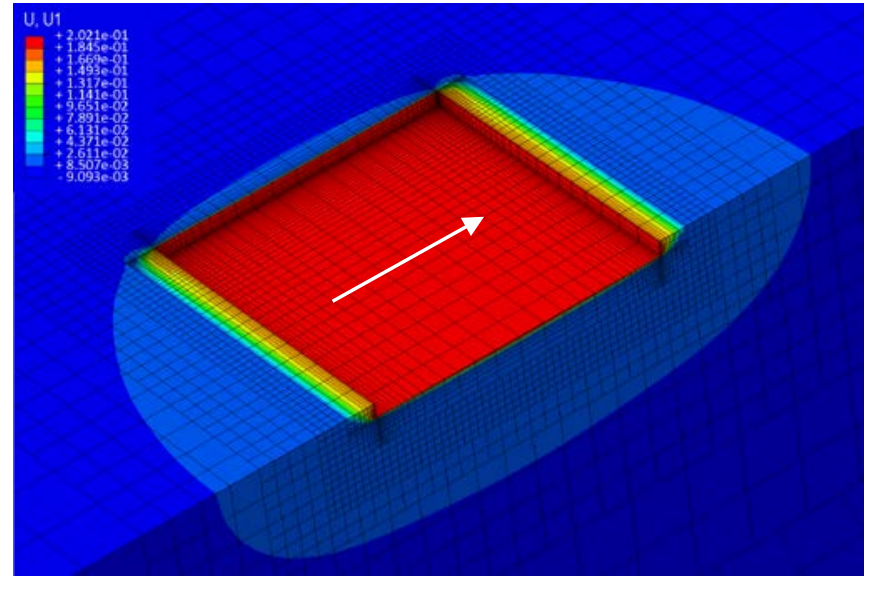

(a)

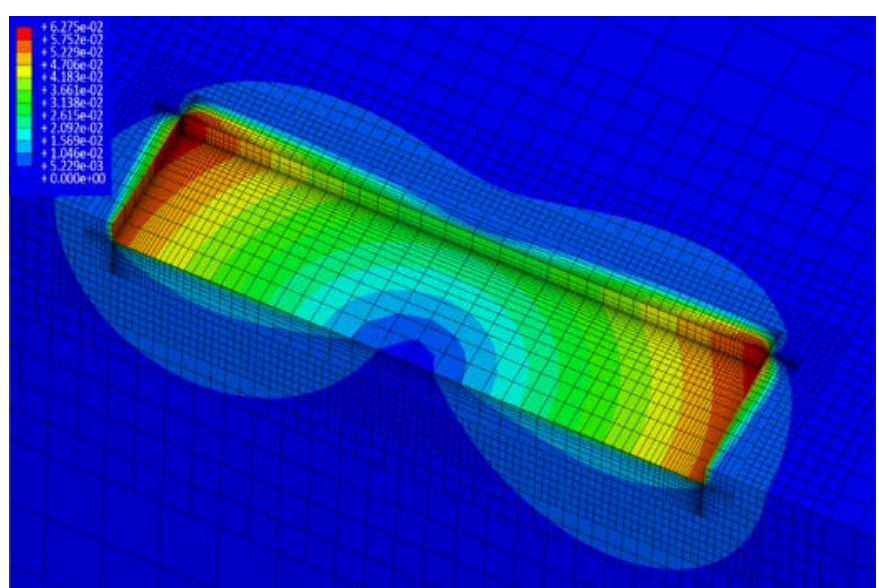

(b)

Figure 10. Contours of plastic displacement for cross section of the model under (a) pure translation and (b) pure torsion 


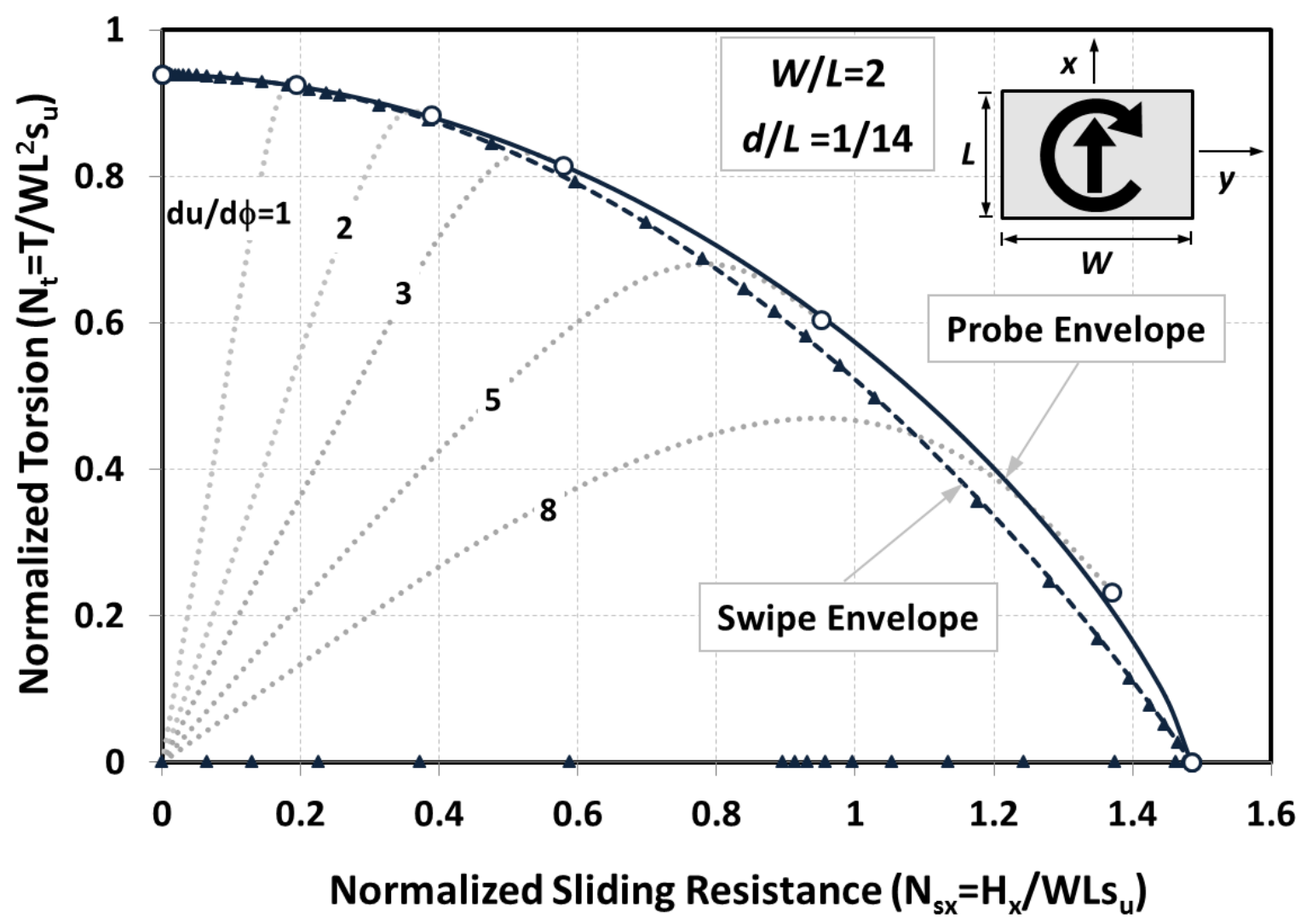

Figure 11. FEA yield envelope for combined shear $x$-torsion loading 


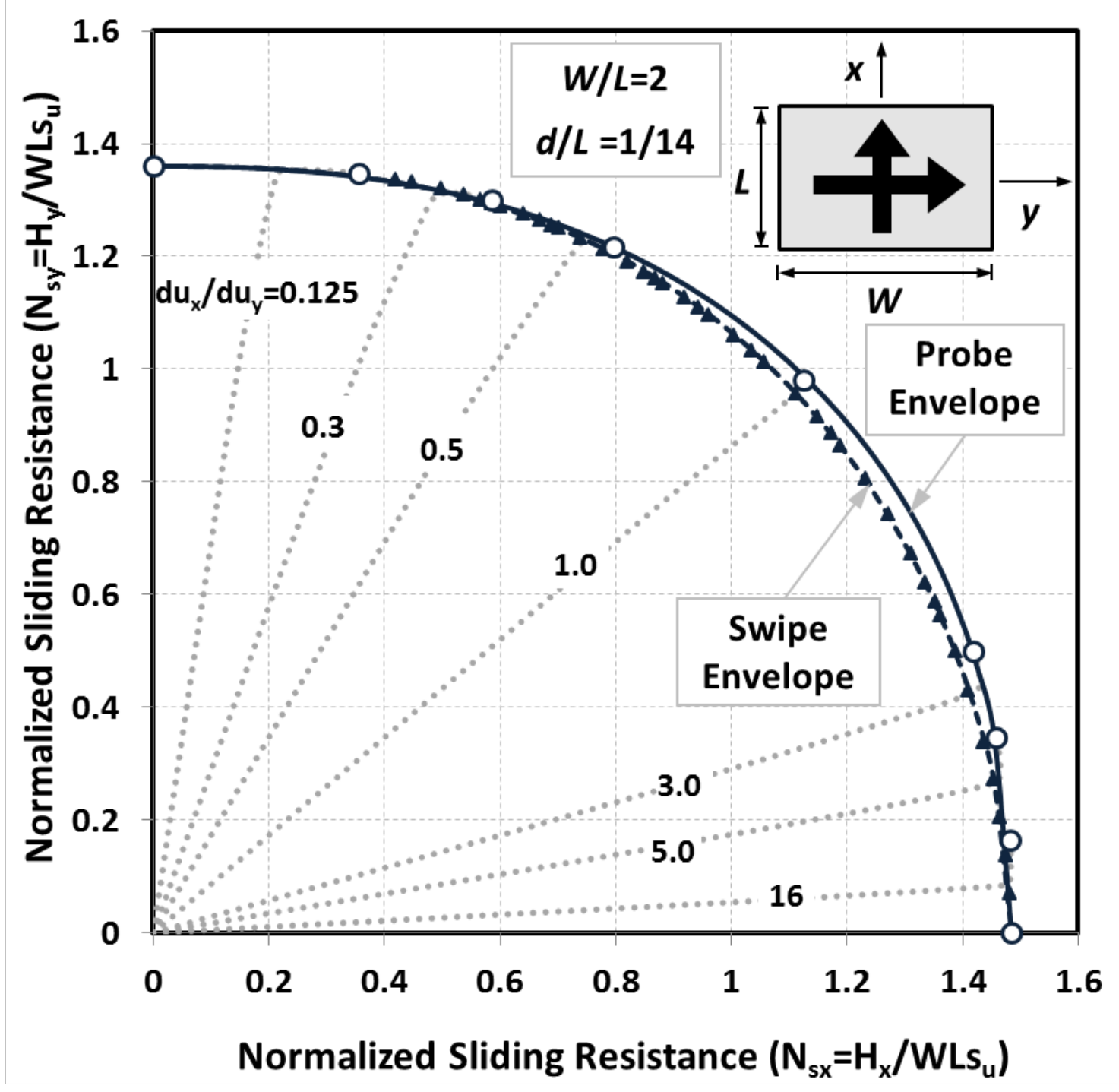

Figure 12. FEA yield envelope for combined $x-y$ translational loading 


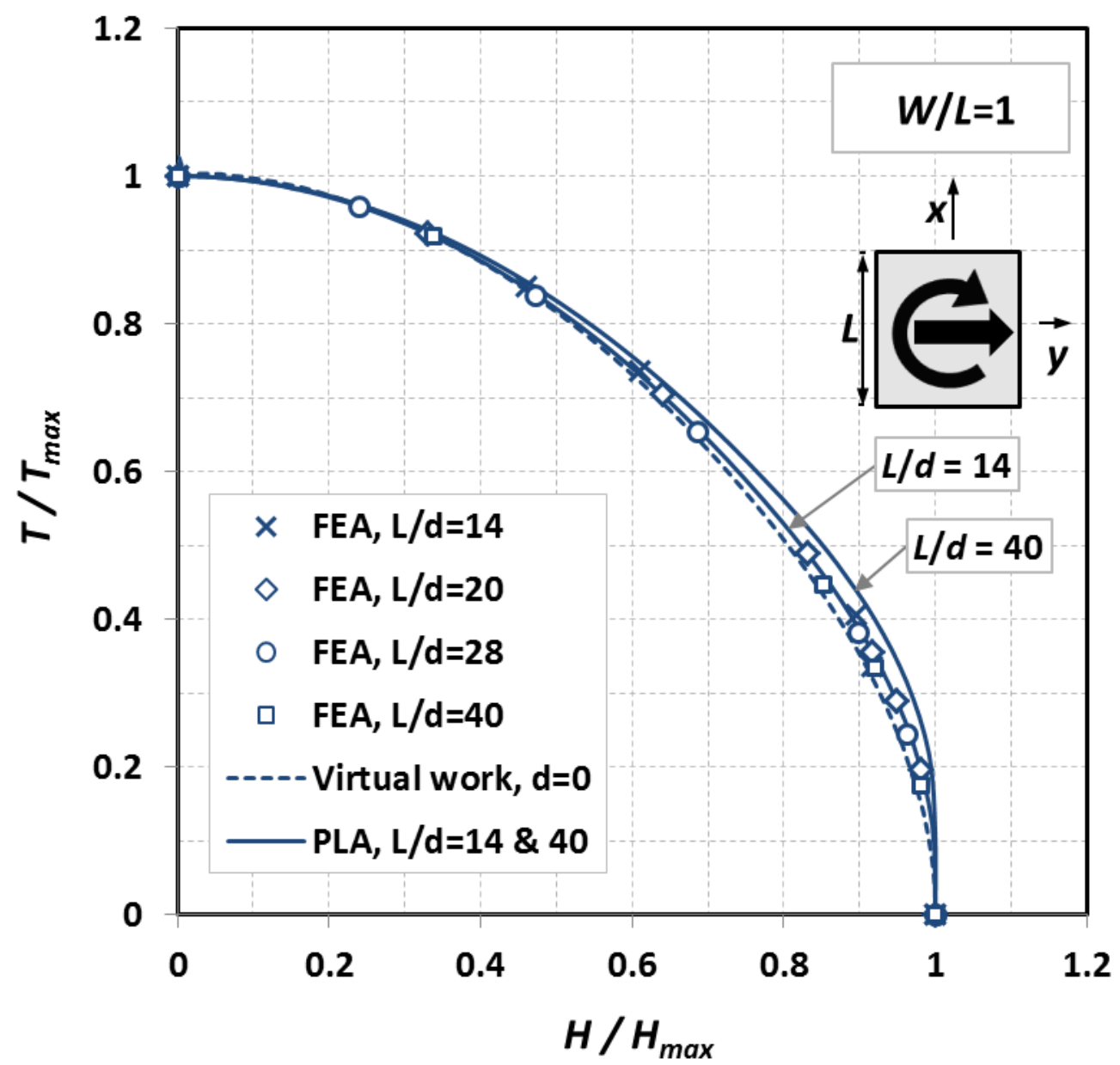

Figure 13. Comparisons of shear-torsion predictions for square footing 


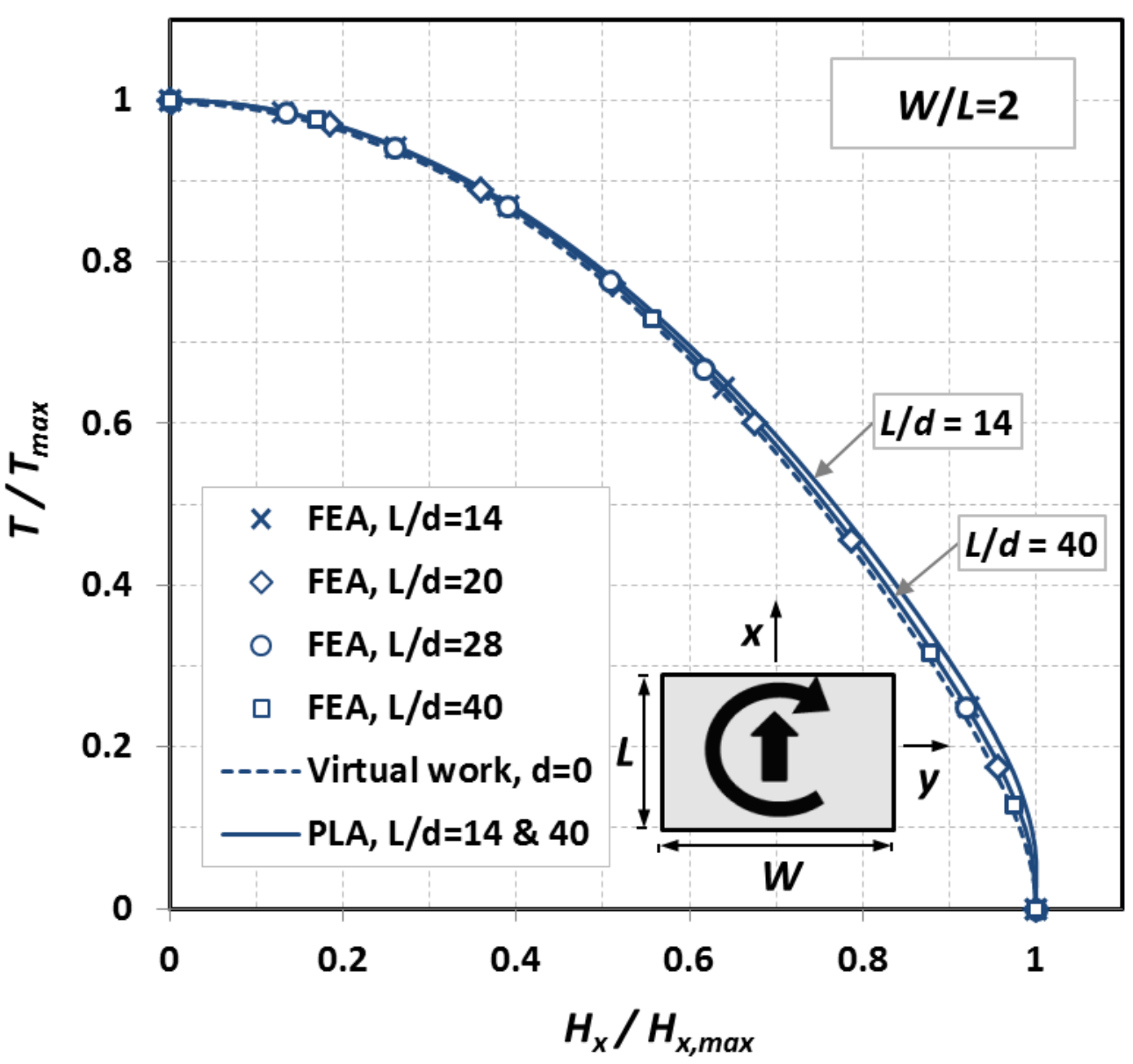

Figure 14. Comparisons of shearx-torsion predictions for rectangular footing 


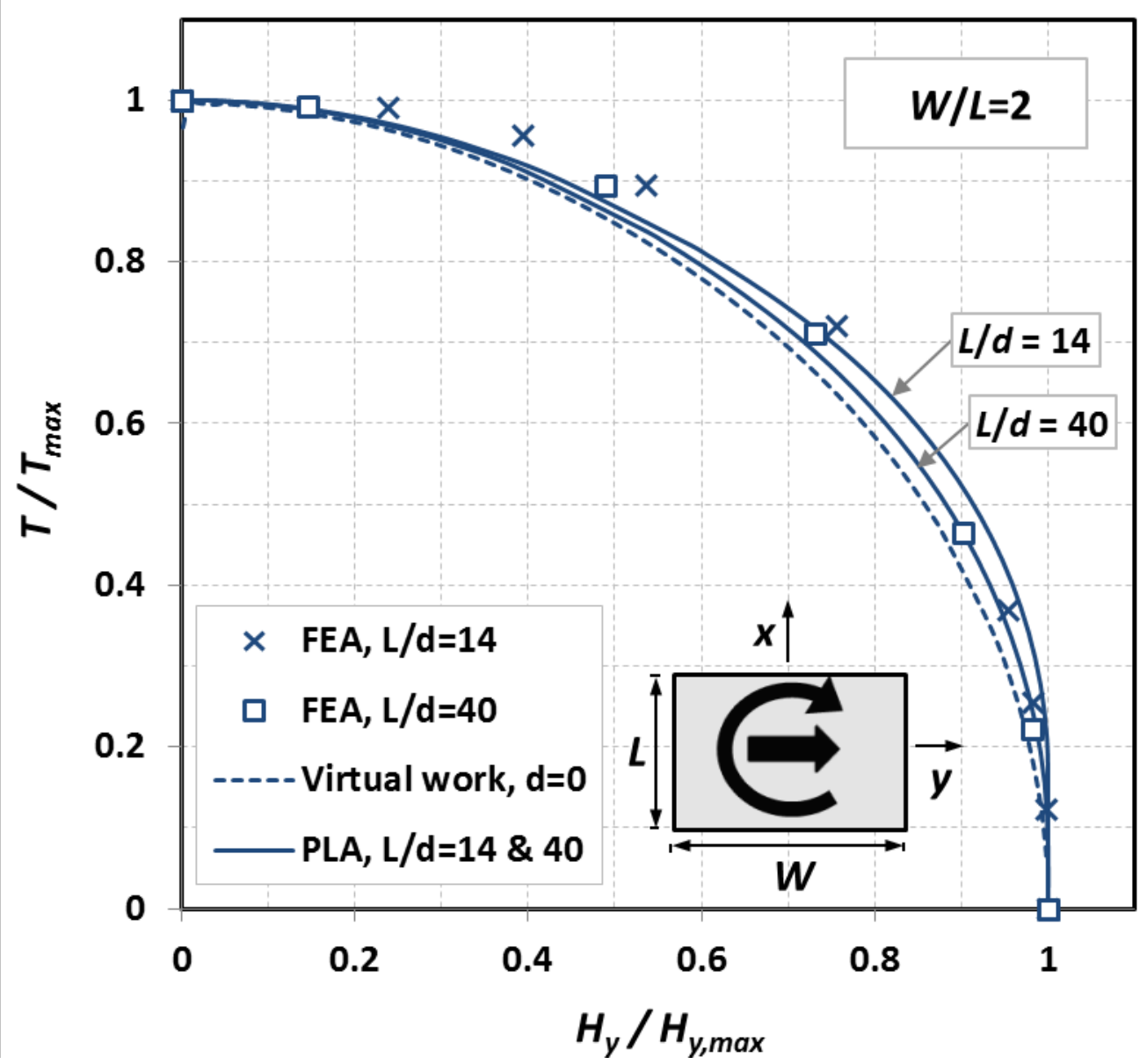

Figure 15. Comparisons of sheary-torsion predictions for rectangular footing 


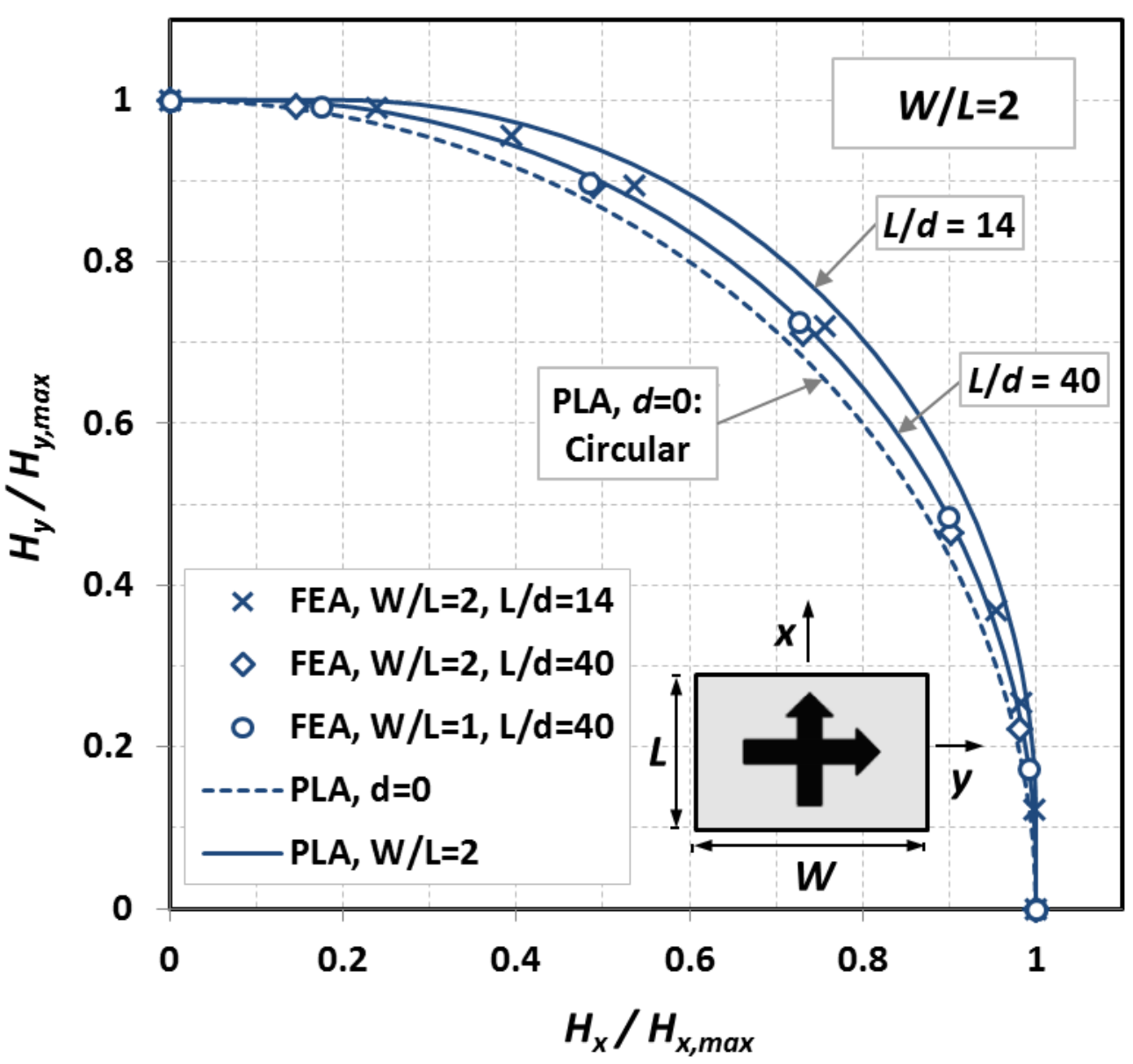

Figure 16. Comparisons of shearx-sheary predictions 


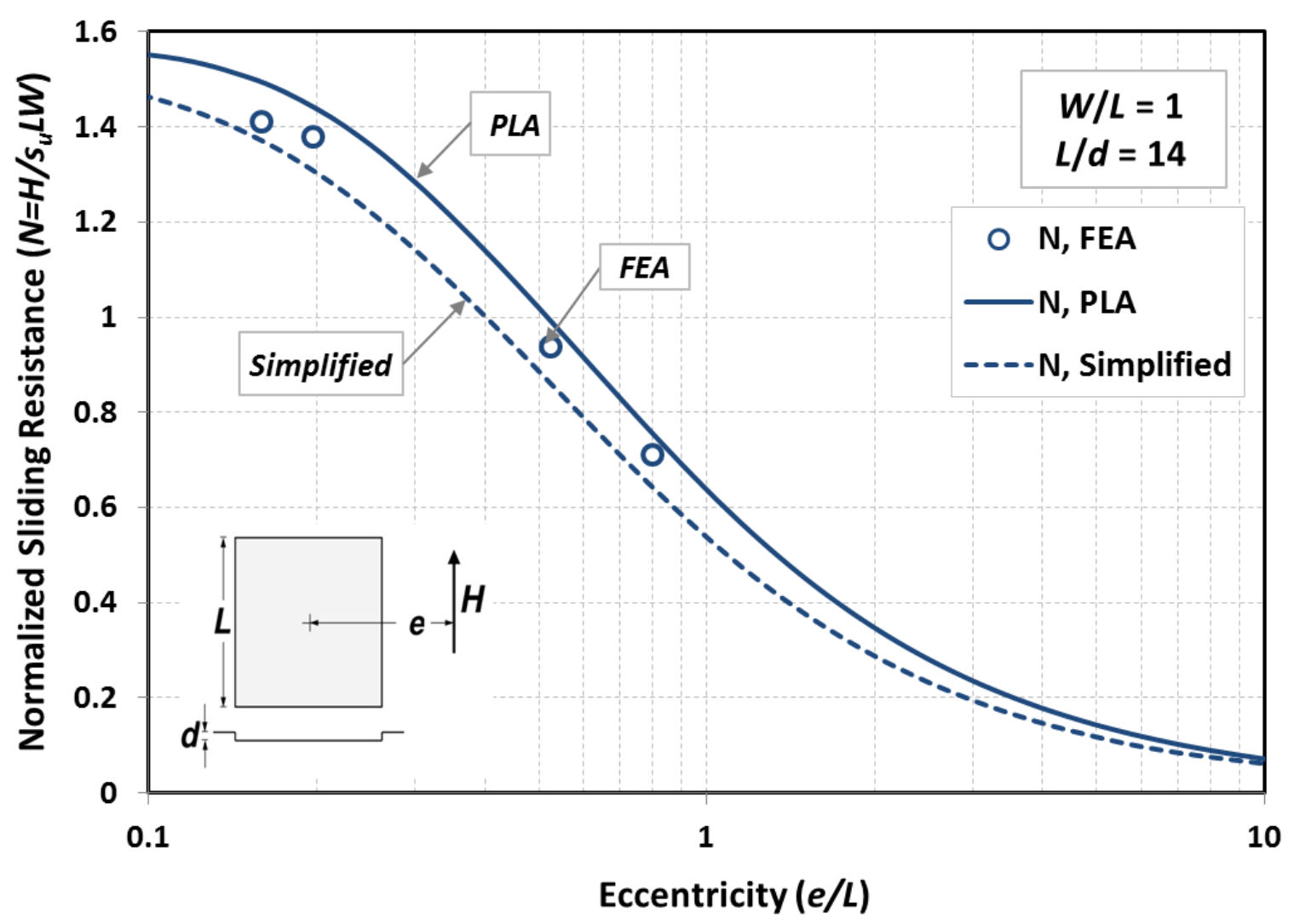

Figure 17. Load capacity reduction versus eccentricity: square footing of $L / d=14$ 


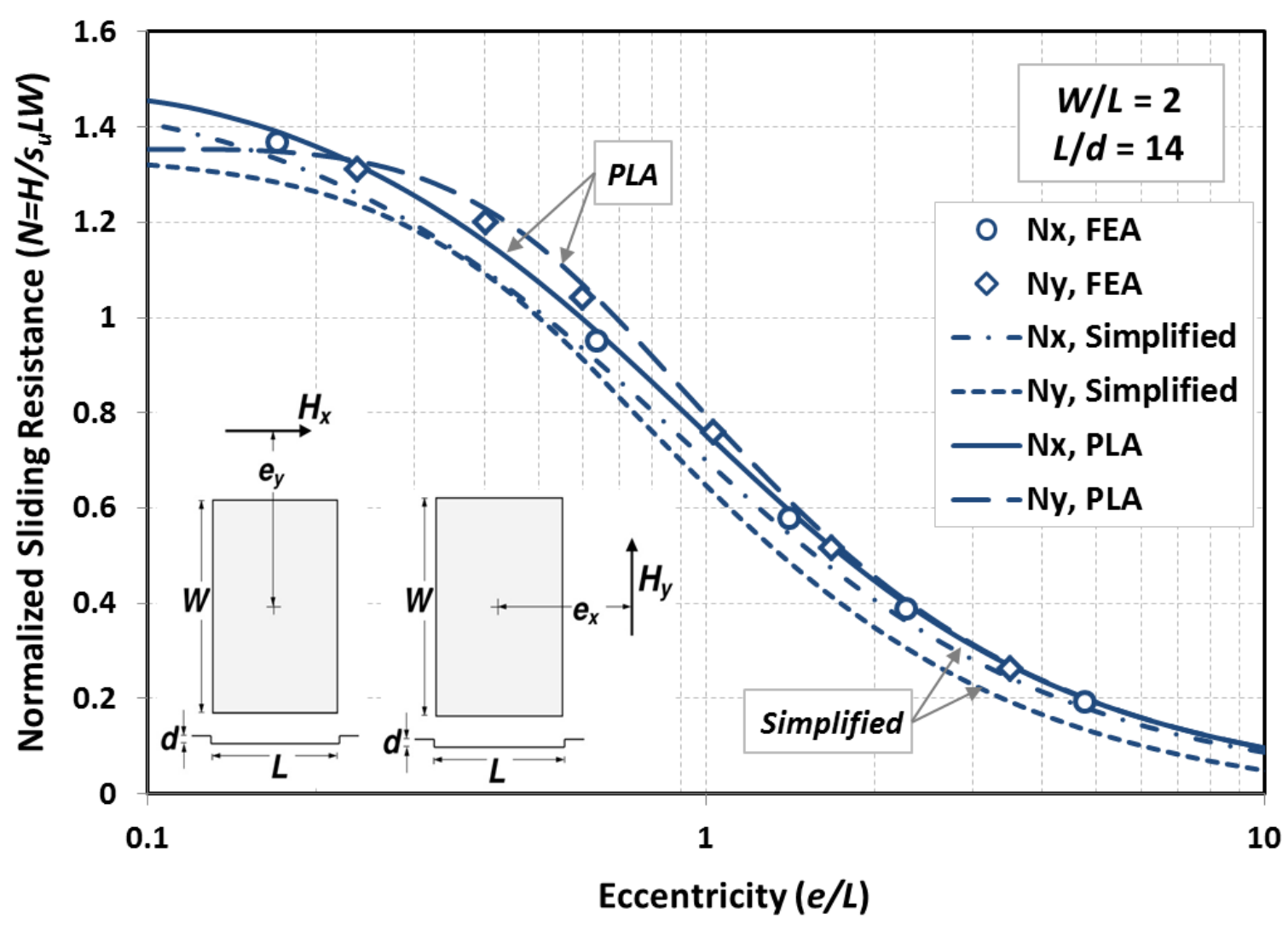

Figure 18. Load capacity reduction versus eccentricity: $L / W=2$ rectangular footing of $L / d=14$ 


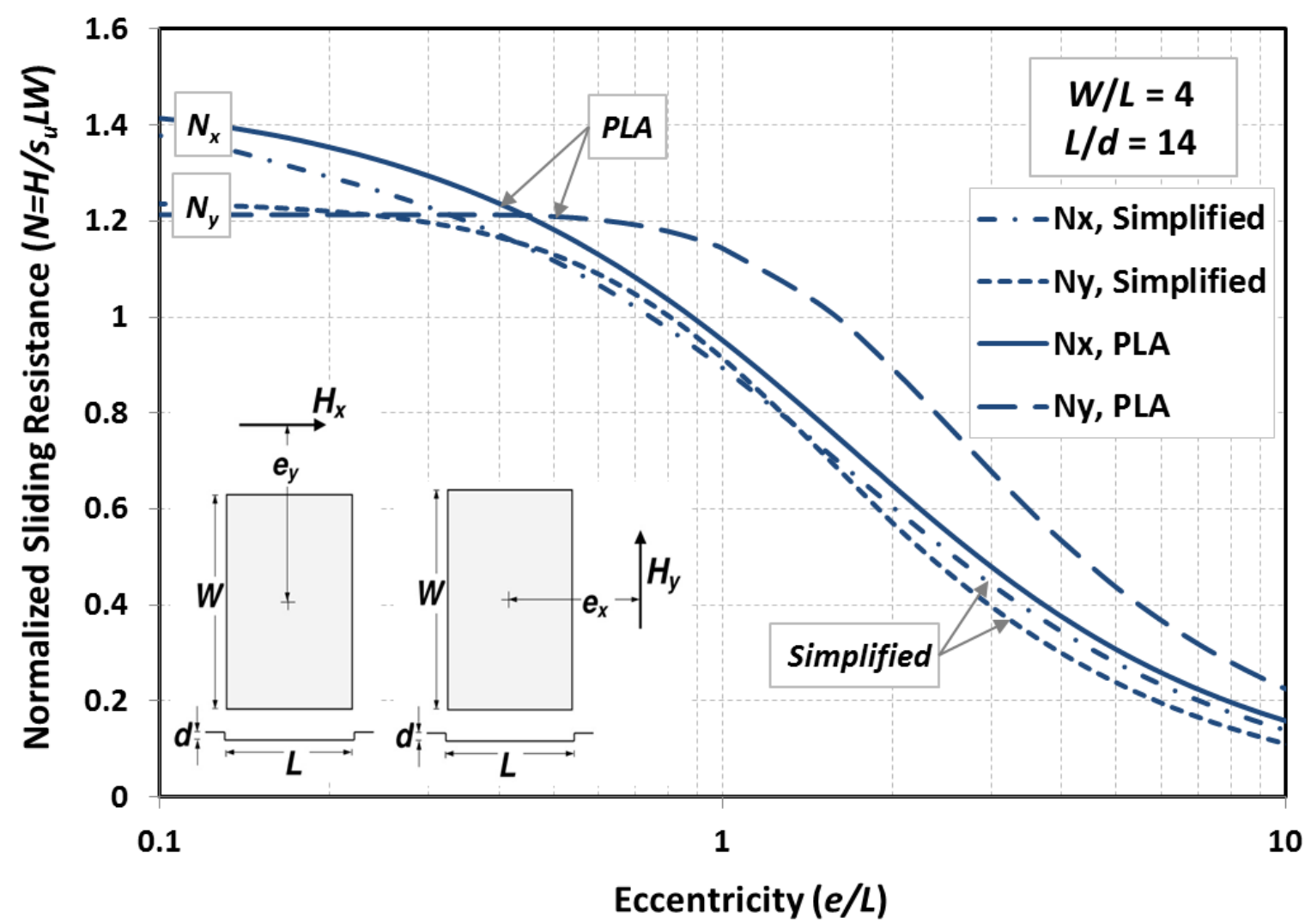

Figure 19. Load capacity reduction versus eccentricity: $L / W=4$ rectangular footing of $L / d=14$ 


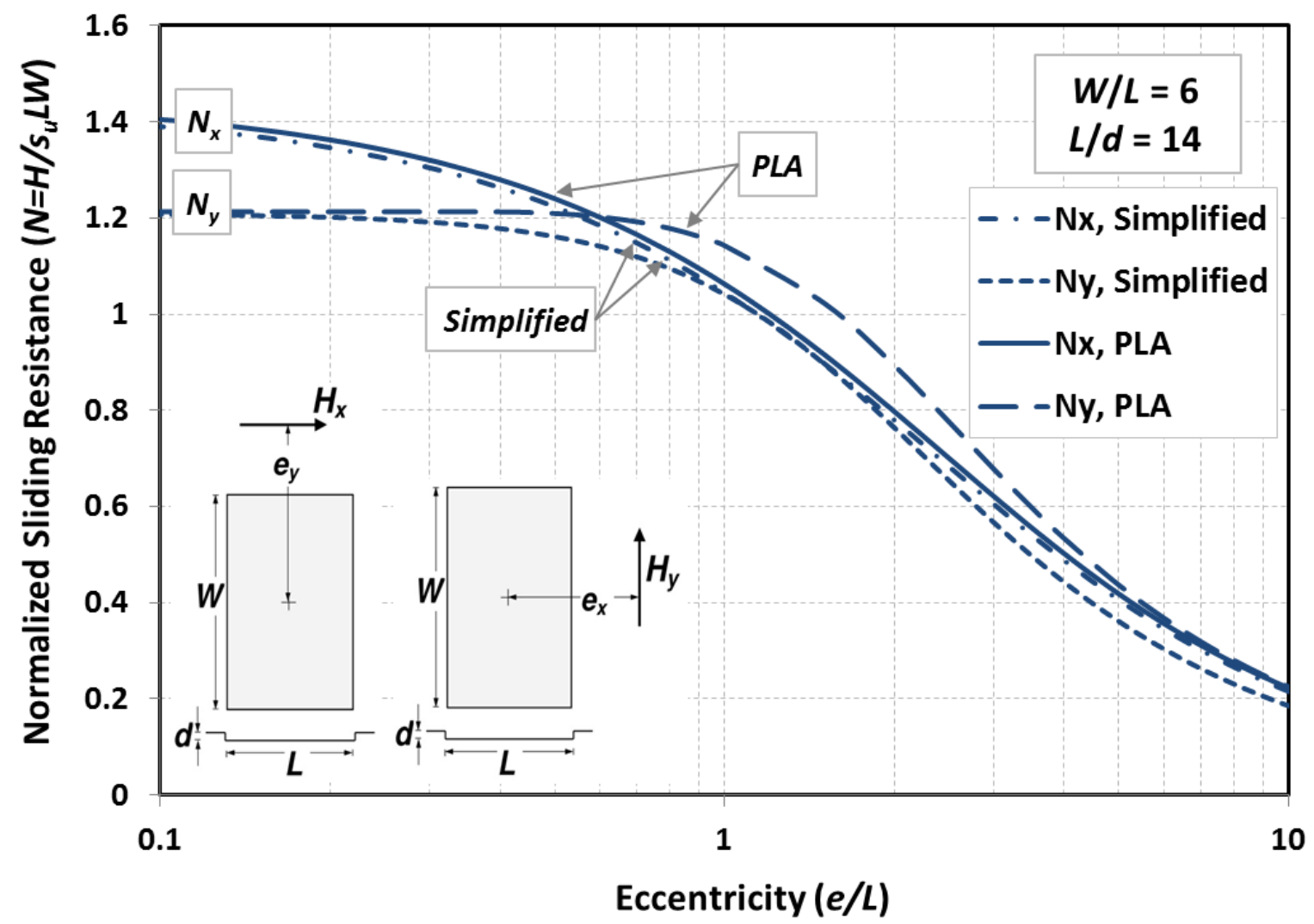

Figure 20. Load capacity reduction versus eccentricity: $L / W=6$ rectangular footing of $L / d=14$ 